\title{
Numerical simulation of detonation initiation and propagation in supersonic combustible mixtures with non-uniform species
}

\author{
Xiaodong Cai ${ }^{\mathrm{a}}$, Jianhan Liang ${ }^{\mathrm{a}, *}$, Ralf Deiterding ${ }^{\mathrm{b}}$, Zhiyong Lin $^{\mathrm{a}}$ \\ ${ }^{a}$ Science and Technology on Scramjet Laboratory, National University of Defense Technology, \\ 410073, Hunan Changsha. \\ ${ }^{\mathrm{b}}$ Aerodynamics and Flight Mechanics Research Group, University of Southampton, Highfield \\ Campus, Southampton SO17 1BJ, United Kingdom
}

\begin{abstract}
Adaptive high-resolution simulations of gaseous detonation using a hot jet initiation were conducted in supersonic combustible mixtures with spatially nonuniform species. The two-dimensional Euler equations were used as the governing equations in combination with a detailed hydrogen-oxygen reaction model. Three different groups of mixtures, which represent various degrees of chemical reactivity, were investigated. The results show that when the mixtures generally have a high degree of chemical reactivity, detonation initiation can eventually be realized successfully by Mach reflection as well as the DDT mechanism, independent of the spatial distribution of the mixture in the channel. A recurring four-stage sequence of detonation initiation, detonation attenuation, initiation failure and detonation reinitiation can be identified. When the mixtures generally have an intermediate degree of chemical reactivity,
\end{abstract}

Corresponding author. Email addresses: cai-chonger@hotmail.com, jhleon@vip.sina.com, r.deiterding@soton.ac.uk, linzy96@nudt.edu.cn 
detonation combustion can be fully realized in the channel, where different degrees of overdrive are found in the upper lower half. After the shutdown of the hot jet, the overdriven detonation attenuates gradually and eventually a slightly overdriven detonation and a slightly underdriven detonation are generated, which can be regarded as a new stable state of propagation. However, whether a detonation can be initiated successfully is determined by the spatial mixture distribution. In mixtures with low degree of chemical reactivity, detonation initiation can generally not be realized. In this case, successful realization of detonation initiation should be realizable by using of a stronger hot jet.

Key words: detonation combustion, hot jet initiation, supersonic combustible mixtures, non-uniform species, chemical reactivity

\section{Nomenclature}

$f_{M_{1}}=$ The overdrive degree for the mixture $\mathrm{M}_{1}$;

$f_{M_{2}}=$ The overdrive degree for the mixture $\mathrm{M}_{2}$;

$l_{i g}=$ The induction length of one-dimensional ZND model; mm

$M_{1}=$ The mixture in the lower half of the domain;

$\mathrm{M}_{2}=$ The mixture in the upper half of the domain;

$M a_{\infty}=$ Mach number of the incoming flow;

$P t s / l_{i g}=$ The number of the grid points distributed in the induction length;

$R=$ The gas constant;

$r_{l}=$ The refinement ratio of the refinement level $l$; 
$\Delta T=$ The oscillating period; $\mu \mathrm{s}$

$V_{C J}=$ The Chapman-Jouguet velocity; $\mathrm{m} / \mathrm{s}$

$V_{C J M_{1}}=$ The Chapman-Jouguet velocity for the mixture $\mathrm{M}_{1} ; \mathrm{m} / \mathrm{s}$

$V_{C J M_{2}}=$ The Chapman-Jouguet velocity for the mixture $\mathrm{M}_{2} ; \mathrm{m} / \mathrm{s}$

$\mathrm{X}_{1}=$ The length of the straight channel; $\mathrm{cm}$

$\mathrm{X}_{2}=$ The distance between the hot jet and the head wall; $\mathrm{cm}$

$\mathrm{X}_{3}=$ The width of the hot jet; $\mathrm{cm}$

$\Delta x_{l}=$ The spatial step size of the refinement level $l$;

$\mathrm{Y}_{1}=$ The height of the channel; $\mathrm{cm}$

$\beta=$ The tangent angle of the bow shock;

$\gamma_{\infty}=$ The heat capacity ratio of the initial flow;

AMROC $=$ Adaptive Mesh Refinement Object-oriented C++;

$\mathrm{CJ}=$ Chapman-Jouguet;

DDT $=$ Deflagration to Detonation Transition;

FVM = Finite Volume Method;

$\mathrm{KH}=$ Kelvin-Helmholtz;

SAMR $=$ Structured Adaptive Mesh Refinement;

TVD $=$ Total Variation Diminishing;

ZND = Zel'dovich-von Neumann-Döring;

\section{Introduction}

Scramjet engines have become one of the first choices for hypersonic air-breathing 
propulsion systems because of their superior performance when the Mach number is larger than 5 [1]. Scramjets are nowadays closer to the actual engineering application [2][3], yet their applicability is still limited because of the low net thrust. Compared with the Brayton cycle adopted in scramjet combustors, detonation combustion has a far higher thermodynamic efficiency [4]. The inherent theoretical advantage of detonations has promoted investigations of detonation engines for advanced propulsion. It is therefore indicated that if a detonation wave could be realized in supersonic combustible mixtures in scramjet combustors, scramjet performance might be improved greatly.

Reliable initiation is one of the key issues in detonation investigations. Compared with direct initiation [5-7], which needs large energy, another possibility oice is to use a hot jet that can also realize initiation quickly [8]. Numerous studies have been conducted using a hot jet initiation in quiescent combustible mixtures [9-17], but rather few researches have been carried out in supersonic combustible mixtures. Detonation initiation and propagation using a hot jet were investigated experimentally by Ishii et al. [18] in combustible mixtures whose Mach numbers were 0.9 and 1.2. Han et al. [19, 20] conducted experiments on detonation initiation and DDT process using a hot jet in supersonic combustible mixtures with Mach numbers 3.0 and 4.0, where detonations were initiated through shocks or shock reflections [21-26] induced by the hot jet. A series of numerical simulations on detonation combustion in supersonic hydrogenoxygen mixtures using a hot jet initiation were carried out by Cai et al. [27-29], where the SAMR framework [30, 31] based open-source program AMROC [32-36] was 
utilized. These simulations were conducted using two-dimensional Euler equations both with a simplified reaction model [33] and a detailed reaction model [37].

It should be noted that the experimental and numerical studies [18-20, 27-29] were all conducted in uniform combustible mixtures. Considering the actual flight conditions for hypersonic air-breathing propulsion systems, supersonic incoming flows are normally non-uniform. Therefore, understanding the behavior of detonation initiation and propagation in non-uniform combustible mixtures is important for detonation physics and practical applications. Thomas et al. [38] and Kuznetsov et al. [39] performed experiments on detonation propagation under concentration gradients. They found that the occurrence of transition to detonation depended significantly on the sharpness of concentration gradients, and indicated that smooth concentration gradients tended to assist the transition process while sharp concentration gradients might lead to detonation failure due to the separation of the shock front and reaction zone. Sochet et al. [40] investigated experimentally detonation initiation in combustible mixtures with non-uniform concentration produced by molecular diffusion, gravity and turbulence, and found that detonations could not be observed due to the limit time delay which could lead to a given concentration distribution. Ishii et al. [41] performed experiments on the behavior of detonations in non-uniform mixtures with concentration gradients normal to the propagation direction and showed that a tilted wave front was created, whose angle was consistent with the deflection angle of the detonation front obtained from trajectories of the triple point. Weber et al. [42] studied numerically the formation and development of detonation waves stemming from temperature non-uniformity 
using detailed chemical kinetics. Kim et al. [43] showed that the increase of the temperature gradient in a non-uniform temperature zone resulted in a decreasing mixture temperature in the unburned mixture zone, which could reduce the combustion wave speed. Cai et al. [44] investigated numerically detonation initiation and propagation in supersonic combustible mixtures with non-uniform velocities and reported that a dynamically stable structure made up of a normal Mach detonation and a pure Mach stem was finally generated in non-uniform supersonic combustible mixtures.

In the present study adaptive simulations of a detonation with hot jet initiation are conducted in supersonic combustible mixtures with non-uniform species based on various degrees of chemical reactivity. This work is part of an ongoing research program, aiming at providing information to help improve the overall understanding of detonation initiation and propagation in supersonic combustible mixtures.

The remainder of this paper is organized as follows: the calculation method is presented in Section 2, including the introduction of the mathematical model and the numerical scheme. Results are shown in Section 3, in which a convergence analysis with different mesh resolutions, detonation initiation and propagation in different groups of non-uniform supersonic combustible mixtures are discussed. Section 4 gives a qualitative discussion, and finally Section 5 concludes the paper.

\section{Calculation method}

\subsection{Mathematical Model}


Numerical simulations are conducted in a straight channel, as depicted in Fig.1. Reflecting boundaries with slip wall conditions are used on the upper and lower wall, except that a small inflow is embedded into the lower wall which models a hot jet. The right boundary models the inflow condition and the left one the outflow condition. Numerical simulations [45] and experimental observations [46-48] indicate the existence of two types of detonation structures, which are usually classified as regular (weakly unstable) and irregular (unstable) detonations based on the regularity of the cellular structure [49-55]. Self-sustaining CJ detonations in low-pressure hydrogenoxygen mixtures with a high-argon dilution are ideal candidates for detonation simulations in supersonic combustible mixtures as regular detonation cell patterns can be generated [56]. The channel consists of two different kinds of mixtures entering from the right boundary at the same velocity. The mixture of $\mathrm{O}_{2} / \mathrm{H}_{2} / \mathrm{Ar}$ with the molar ratio 1:2:7 under pressure $6.67 \mathrm{kPa}$ and temperature $298 \mathrm{~K}$ at the velocity of $V_{C J}\left(V_{C J}=1627\right.$ $\mathrm{m} / \mathrm{s}$ ) is adopted as a basic example. Another mixture of $\mathrm{O}_{2} / \mathrm{H}_{2} / \mathrm{Ar}$ has the same condition with the basic one, except for a different molar ratio. Here $\mathrm{M}_{1}$ and $\mathrm{M}_{2}$ are used to represent the two mixtures with different molar ratios.

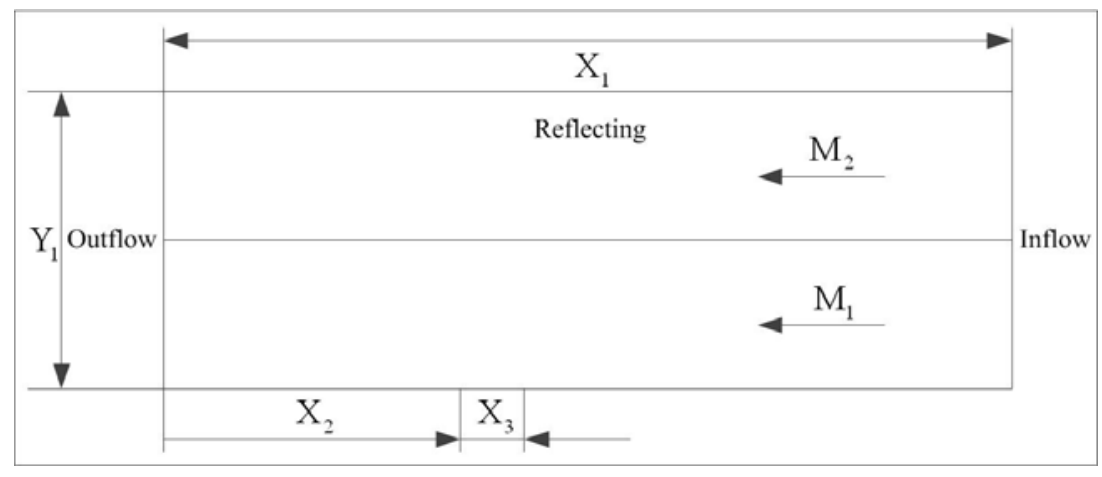

Fig.1 Schematic of the computational setup

When dealing with the inflow condition of the hot jet, the parameter "time" is 
considered to control the duration of the hot jet injection. When the hot jet is shut down, the inflow condition switches to the reflecting condition immediately. As shown in Table 1, the equilibrium CJ state of $\mathrm{H}_{2} / \mathrm{O}_{2}$ with a molar ratio of $2: 1$ under pressure 6.67 $\mathrm{kPa}$ and temperature $298 \mathrm{~K}$ is set to the parameters of the hot jet, which is calculated with Cantera [57].

Table 1 The equilibrium CJ state of the hot jet. Note that the nine species values are given as mass fractions.

\begin{tabular}{ccc}
\hline State parameter & Value & Unit \\
\hline Pressure & 113585.12 & $\mathrm{~Pa}$ \\
Temperature & 3204.8374 & $\mathrm{~K}$ \\
Density & 0.05959 & $\mathrm{~kg} / \mathrm{m}^{3}$ \\
Velocity & 1229.9015 & $\mathrm{~m} / \mathrm{s}$ \\
Energy & 83445.813 & $\mathrm{~J} / \mathrm{m}^{3}$ \\
$\mathrm{H}_{2}$ & 0.024258141648492 & \\
$\mathrm{H}$ & 0.007952664033931 & \\
$\mathrm{O}$ & 0.055139351559790 & \\
$\mathrm{O}_{2}$ & 0.124622185271180 & \\
$\mathrm{OH}$ & 0.161144120322560 & \\
$\mathrm{H}_{2} \mathrm{O}$ & 0.626759466258162 & \\
$\mathrm{HO}_{2}$ & 0.000117215557650 & \\
$\mathrm{H}_{2} \mathrm{O}_{2}$ & 0.000006855348235 & \\
$\mathrm{Ar}$ & 0 & \\
\hline
\end{tabular}

\subsection{Numerical scheme}

Two-dimensional Euler equations with the detailed reaction model are used as the governing equations [32]. A second-order accurate MUSCL-TVD FVM is adopted for convective flux discretization. The hydrodynamic solution process in AMROC is divided into the two steps of numerical flux calculation and reconstruction. Rather than 
the second-order accurate Strang splitting, the first-order accurate Godunov splitting is adopted for considering the source term as almost the same performance is achieved with higher computational efficiency [32]. A hybrid Roe-HLL [32] Riemann solver is used to construct the inter-cell numerical upwind fluxes while the Van Albada limiter with MUSCL reconstruction is applied to construct a second-order method in space. The MUSCL-Hancock technique [58] is adopted for second-order accurate time integration.

Since the inviscid equations are used, the only source of diffusion is due to the numerical scheme and its magnitude determined by grid resolution [59]. Yet, even when solving the viscous equations at low grid resolution, numerical diffusion dominates over the physical one, cf. Samtaney and Pullin [60] for an excellent discussion of this issue. However, even at high grid resolution qualitative agreement is obtained in detonation simulations both by solving Euler and Navier-Stokes equations, especially for regular detonations. Previously Oran et al. [61] performed a series of detonation simulations using both Euler and Navier-Stokes equations with detailed chemical kinetics. They observed similar structures of regular detonations for both Euler and Navier-Stokes equations, and indicated that the small-scale structures that are eliminated in Euler computations do not affect the overall features of regular detonations. Very recently Mazaheri et al. [62] and Mahmoudi et al. [63, 64] showed that from the comparison of detonations solved both by Euler and Navier-Stokes equations, diffusion effect has no crucial role in the overall structure of regular detonations due to the negligible effect of hydrodynamic instabilities. Therefore, the 
results obtained in this paper using Euler equations for regular detonations are nevertheless expected to give at least qualitatively correct conclusions.

\section{Results and analysis}

As shown in Fig.1, the length of the straight channel varies from $X_{1}=12 \mathrm{~cm}$ to $X_{1}$ $=16 \mathrm{~cm}$, while the height is fixed with $\mathrm{Y}_{1}=3 \mathrm{~cm}$. The distance between the hot jet and the head wall is $X_{2}=4.5 \mathrm{~cm}$, and the width of the hot jet is $X_{3}=0.4 \mathrm{~cm}$. The initial mesh resolution in both directions is $2.5 \times 10^{-4} \mathrm{~m}$, and the induction length for the basic mixture is $l_{i g}=1.509 \mathrm{~mm}$, calculated with Cantera. For the five-level refinement with the corresponding refinement factors $r_{1}=2, r_{2}=2, r_{3}=2, r_{4}=2$ adopted, the highest resolution can be as high as $96.8 \mathrm{Pts} / \mathrm{l}_{\text {ig }}$, which is eight times higher resolved than that in [61] and two times higher than that in $[29,54]$. The computations are conducted on a cluster using 120 Intel E5-2692 2.20 GHz (Ivy Bridge) processors. The refinement factor is the ratio between the spatial steps $\Delta x_{l}$ and $\Delta x_{l-1}$ of levels $l$ and $l-1$, respectively, i.e. $r_{l}=\Delta x_{l-1} / \Delta x_{l}$.

As shown in Table 2, three different groups of mixtures are employed in total in this study. All mixtures are chosen based on the basic example of Section 2.1. The three groups of mixtures can represent three degrees of chemical reactivity based on three different average molar ratios, i.e. G1: 1:2:3.5; G2: 1:2:7; G1: 1:2:10.5. In addition, positions for two different mixtures in the same group are also interchanged with one another to investigate whether the mixture distributions also play a significant role.

Table 2 Details of three different groups of stoichiometric $\mathrm{O}_{2} / \mathrm{H}_{2} / \mathrm{Ar}$ mixtures. 


\begin{tabular}{c|c|c}
\hline Group & \multicolumn{2}{|c}{ Molar Ratio } \\
\hline G1 & G1.1: $\mathrm{M}_{1}=1: 2: 7, \mathrm{M}_{2}=1: 2: 0$ & G1.2: $\mathrm{M}_{1}=1: 2: 0, \mathrm{M}_{2}=1: 2: 7$ \\
G2 & G2.1: $\mathrm{M}_{1}=1: 2: 3.5, \mathrm{M}_{2}=1: 2: 10.5$ & G2.2: $\mathrm{M}_{1}=1: 2: 10.5, \mathrm{M}_{2}=1: 2: 3.5$ \\
G3 & G3.1: $\mathrm{M}_{1}=1: 2: 7, \mathrm{M}_{2}=1: 2: 14$ & G3.2: $\mathrm{M}_{1}=1: 2: 14, \mathrm{M}_{2}=1: 2: 7$ \\
\hline
\end{tabular}

\subsection{Convergence analysis}

The mixture group G1.1 is adopted here for the investigation of numerical convergence. For regular detonations, an effective resolution up to $44.8 \mathrm{Pts} / \mathrm{l}_{\text {ig }}$ was used in previous two-dimensional detonation simulations with a detailed reaction model $[29,32,54]$, which indicates that this resolution is sufficient for resolving reliably even the secondary triple points.

Here, three different mesh refinements are shown in Fig.2, and the highest resolution in Fig.2(a) is $48.4 \mathrm{Pts} / \mathrm{l}_{i g}, 96.8 \mathrm{Pts} / \mathrm{l}_{\text {ig }}$ in Fig.2(b) and $193.6 \mathrm{Pts} / \mathrm{l}_{\text {ig }}$ in Fig.2(c), respectively, which are all larger than $44.8 \mathrm{Pts} / \mathrm{l}_{i g}$. Overall, the same pattern of Mach reflection, slip line (shear layer) due to KH instabilities, bow shock, and shockinduced combustion is observed at all three resolutions, and the flow structures are always well resolved within the highest level (shown in red). It is very important for the setting of refinement thresholds to enable adequate coverage of shock wave and combustion zone and their surrounding regions in detonation simulations because the interaction with refinement boundaries could otherwise create artificial numerical disturbances. For the three different resolutions investigated here, cf. Fig.2(a)-(c), the above requirements are always satisfied. Eventually, as a compromise between numerical resolution and computational cost, the second highest resolution with five levels, cf. Fig.2(b), is chosen as the configuration for all the subsequent simulations. 

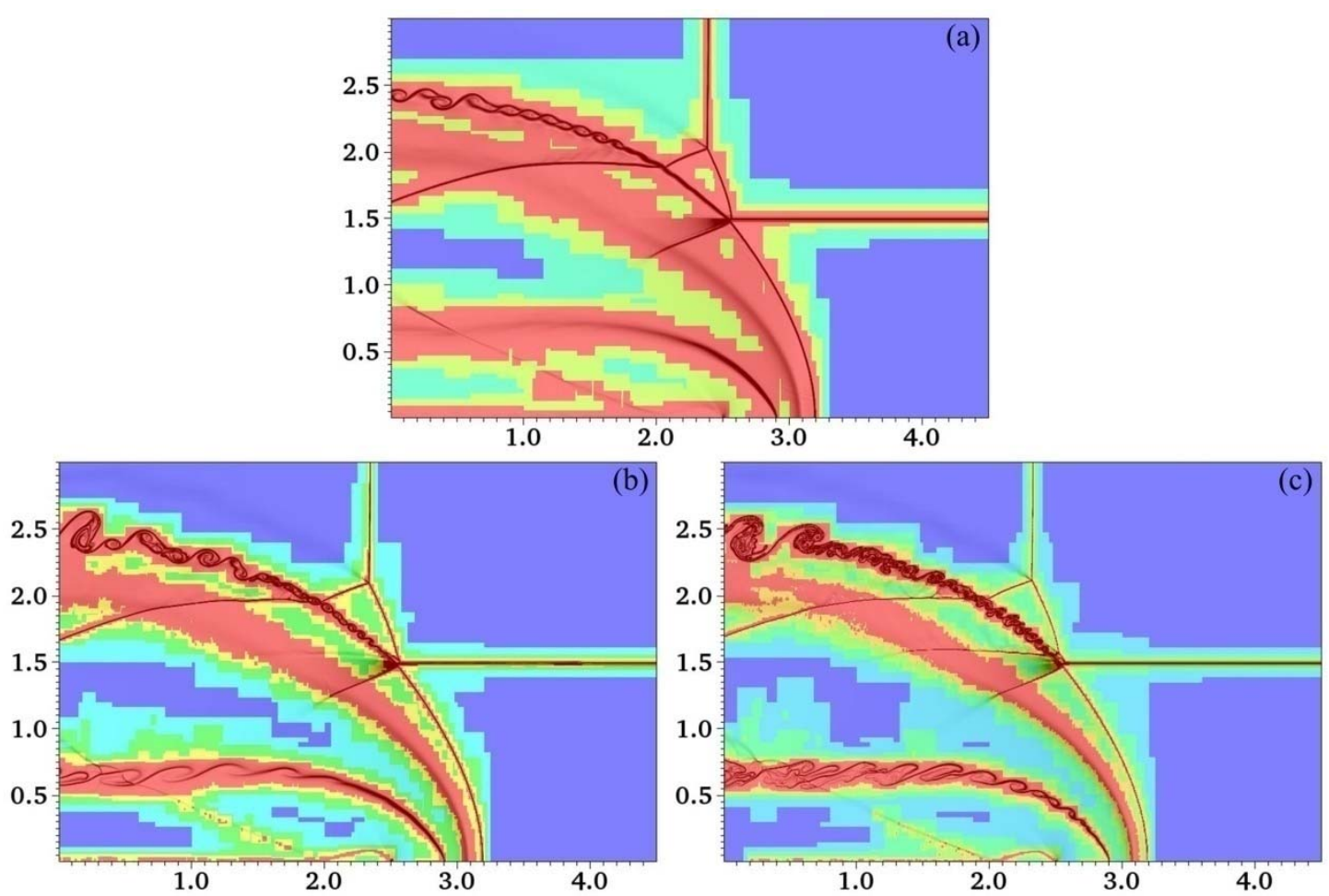

Fig.2 Distributions of three different mesh refinements: (a) four levels of $r_{1}=2, r_{2}=2$,

$$
\begin{gathered}
r_{3}=2 \text {; (b) five levels of } r_{1}=2, r_{2}=2, r_{3}=2, r_{4}=2 \text {; (c) six levels of } \\
r_{1}=2, r_{2}=2, r_{3}=2, r_{4}=2, r_{5}=2 .
\end{gathered}
$$

\subsection{Results for Mixture Group G1}

\subsubsection{Mixture Group G1.1}

After the injection of the hot jet into the channel, a bow shock is induced quickly. The bow shock becomes stronger gradually and reaches the interface between the two different mixtures with different densities. When it crosses through the interface, the bow shock is bending toward the upper half part, and a corner is formed on the interface, as shown in Fig.3(a). According to the Rankine-Hugoniot equation, the strength of the bow shock is $\frac{p-p_{\infty}}{p_{\infty}}=\frac{2 \gamma_{\infty}}{\gamma_{\infty}+1} M a_{\infty}^{2} \sin ^{2} \beta-\frac{2 \gamma_{\infty}}{\gamma_{\infty}+1}\left(M a_{\infty}=V_{C J} / \sqrt{\gamma_{\infty} R T}\right)$, showing that the strength of the bow shock is decided both by the parameters $\gamma_{\infty} R\left(\gamma_{\infty} R\right.$ is 
determined by the characteristics of the mixtures) and the tangent angle $\beta$ of the bow shock. The bow shock bends into the upper half, which can gradually increase the tangent angle $\beta$, thus resulting in pressure matching in the two different mixtures behind the bow shock. Because of the confinement of the channel, the bow shock finally reaches the upper wall and a Mach reflection is generated, as shown in Fig.3(b). However, behind this Mach stem no $\mathrm{OH}$ radicals exist, which is different from the result in [44]. Although $M_{2}$ in the upper half part is more chemically active than $M_{1}$ in the lower half part, behind the Mach stem no reaction is induced.
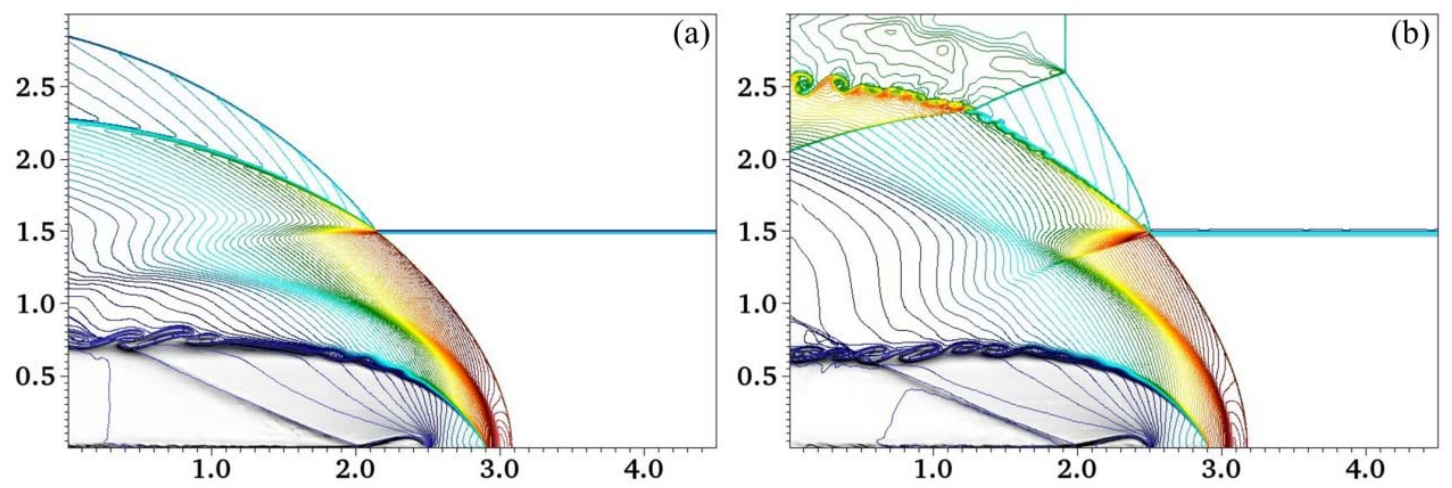

Fig.3 Isolines of density and $\mathrm{OH}$ numerical schlieren images showing the formation of the bow shock and Mach reflection after the hot jet injection. (a) $t=29.45 \mu \mathrm{S}$; (b)

$$
t=83.79 \mu \mathrm{s} .
$$

The Mach stem propagates forward gradually and ultimately reaches the interface. Then the Mach stem is divided into two parts: one continues to propagate in the upper half and another propagates along the bow shock in the lower half, as shown in Fig.4(a). Because of diffusion effects of the large-scale vortices resulting from $\mathrm{KH}$ instabilities behind the Mach stem, small combustion regions are gradually generated along the vortices. Finally, the channel is filled up with two groups of slip lines that are fully combusted, as shown in Fig.4(b). 

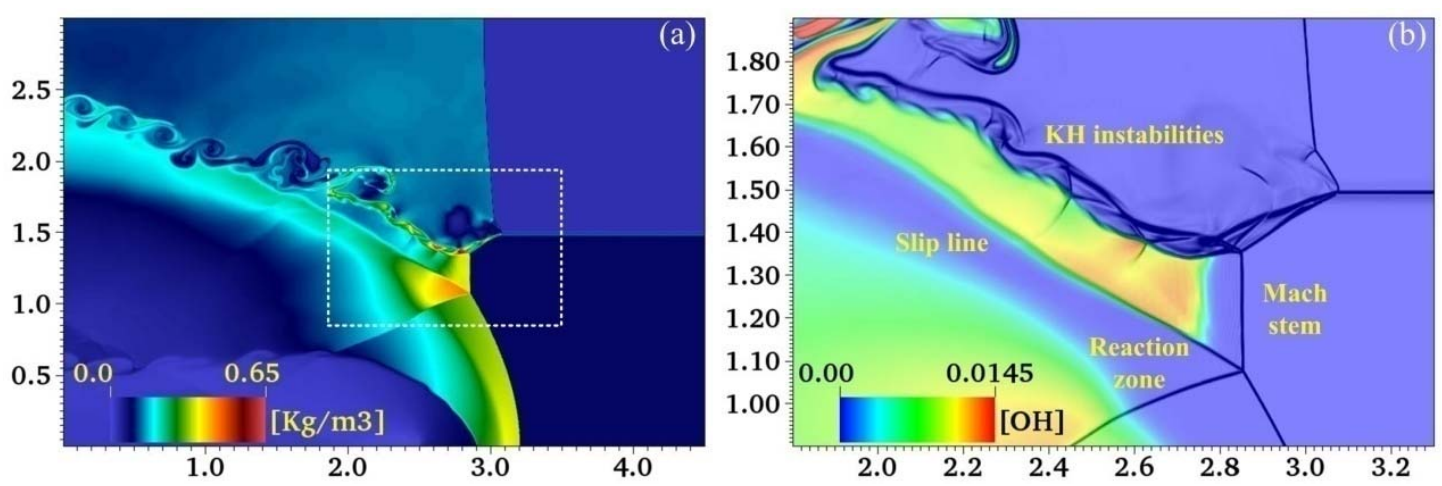

Fig.4 Mach stem as a local detonation at $t=146.82 \mu \mathrm{s}$. (a) is a color plot of density and

(b) is showing a color plot of the $\mathrm{OH}$ mass fraction overlaid by a numerical schlieren image

of the density.

The distance between the Mach stem and the reaction front is $0.86 \mathrm{~mm}$, which is only approximately half of the induction length $\left(l_{i g}=1.509 \mathrm{~mm}\right)$. The reaction front is tightly coupled with the Mach stem, indicating that it is actually a local Mach detonation here. This structure in Fig.4(b) is similar to that when non-uniform velocities are utilized in [44]. However, the difference is that for non-uniform velocities, two parallel slip lines are formed in the middle interface while there is only one slip line generated in the case with non-uniform species. This is due to the fact that the interface in the case with non-uniform species is an approximately normal shock wave, while in the case with non-uniform velocities a curvilinear shock wave is generated in the interface. The formation of a local Mach detonation provides the ignition energy for successful initiation in the lower half part due to the mechanism of triple point collisions [44]. 

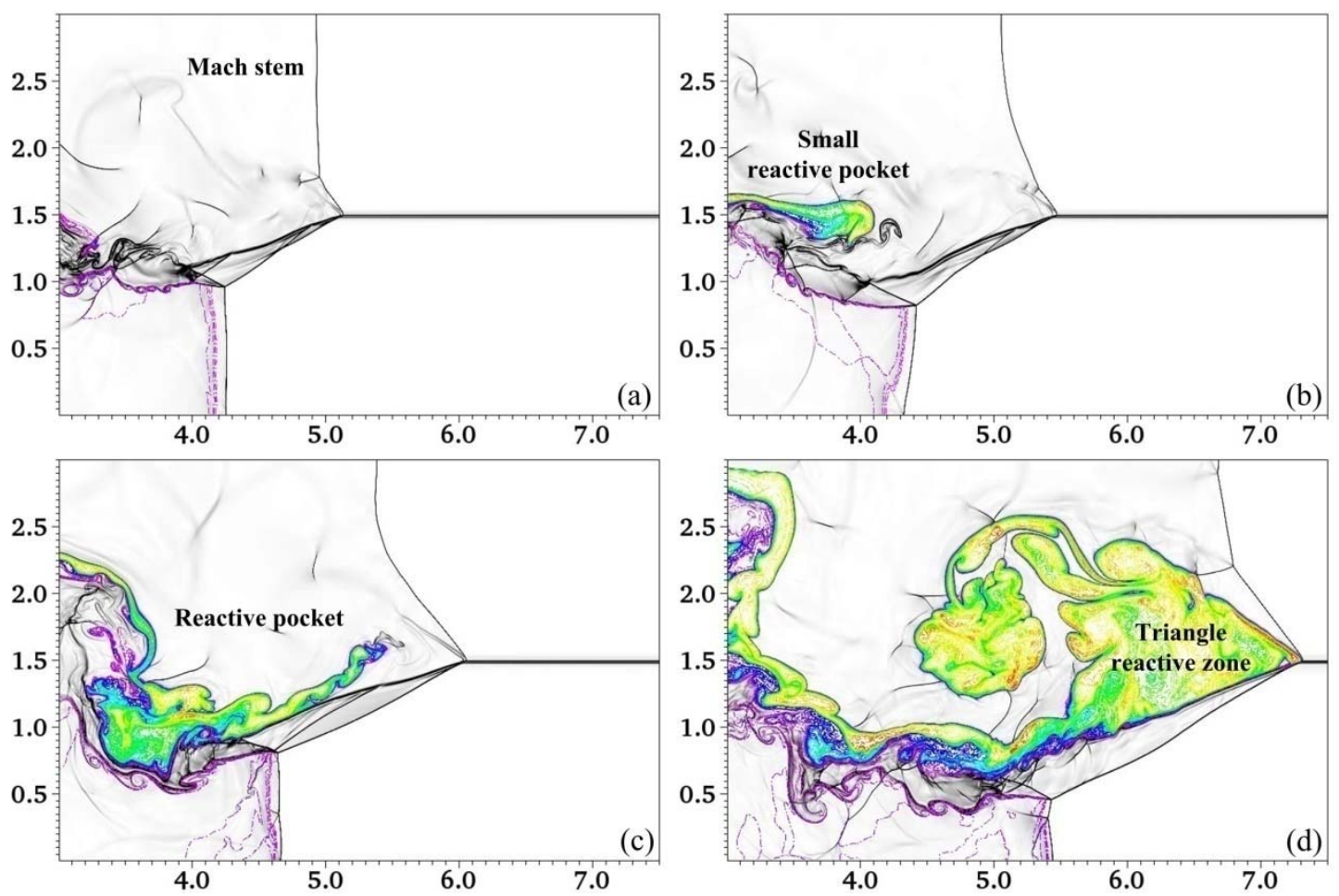

Fig.5 Formation of a triangle-shaped combustion zone resulting from large-scale vortex

interaction along the slip lines (shear layers) depicted by isolines of $\mathrm{OH}$ mass fractions and

numerical schlieren images of density. (a) $t=247.12 \mu \mathrm{s}$; (b) $t=258.12 \mu \mathrm{s}$; (c)

$$
t=272.25 \mu \mathrm{s} \text {; (d) } t=302.35 \mu \mathrm{s} \text {. }
$$

Although initiation has been realized successfully in the mixture $\mathrm{M}_{1}$ in the lower half part, detonation is still not achieved for the mixture $\mathrm{M}_{2}$ in the upper half, where only a pure shock wave is formed, as shown in Fig.5(a). This situation starts to change when the combustion zone far behind the pure shock wave propagates forward along the large-scale vortices and gradually reaches the region right behind the shock wave, as shown in Fig.5(b), (c). Because of the ignition energy provided by the combustion zone, a large triangular combustion zone is formed eventually, as depicted in Fig.5(d). The reaction front behind the shock wave is not tightly coupled with the shock wave, which indicates that the reaction behind the shock wave is actually an oblique shock- 
induced combustion rather than a real detonation.

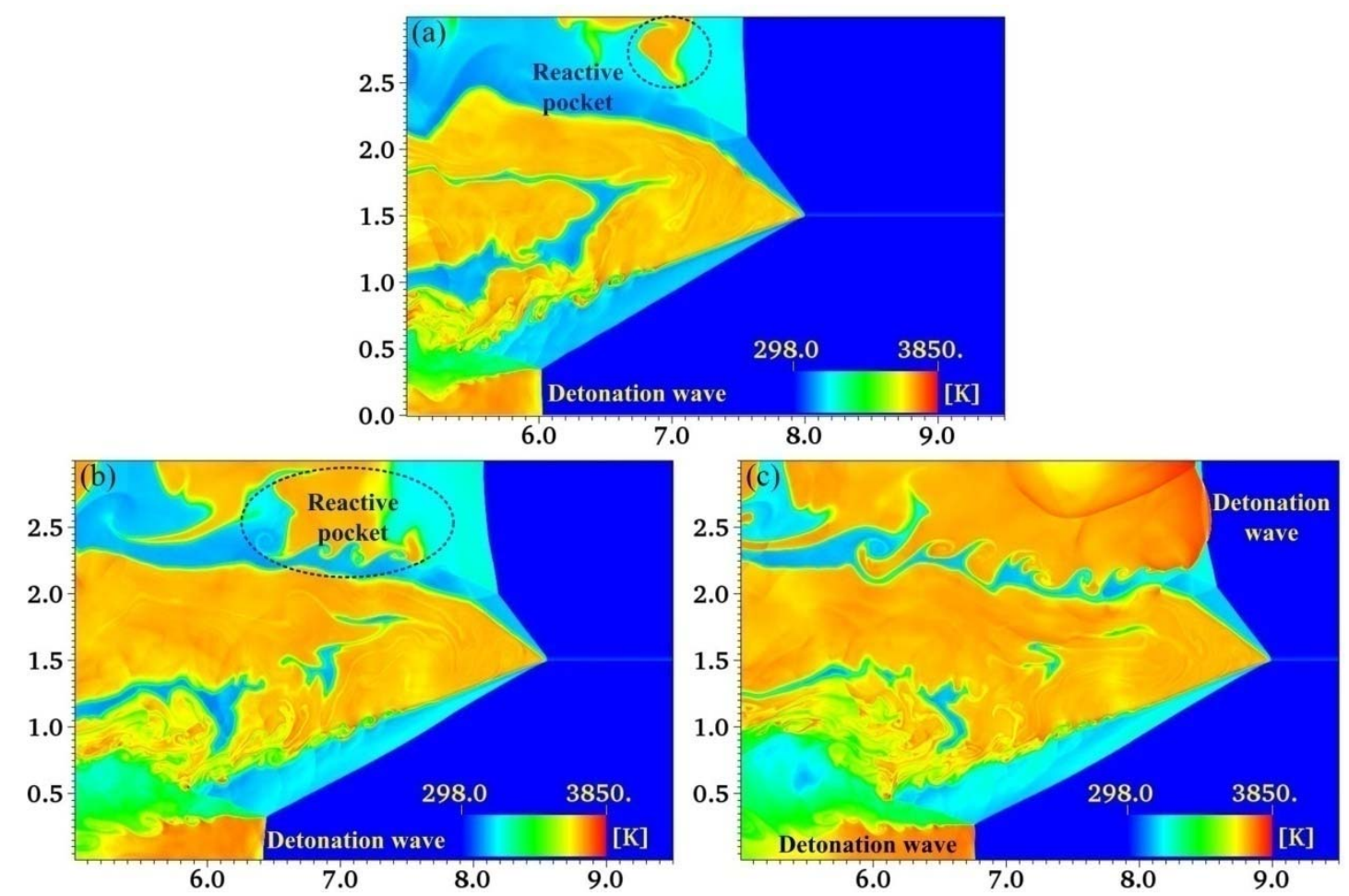

Fig.6 Detonation initiation in the upper half part through the DDT mechanism. (a)

$$
t=316.23 \mu \mathrm{s} \text {; (b) } t=324.67 \mu \mathrm{s} \text {; (c) } t=330.12 \mu \mathrm{s} \text {. }
$$

However, owing to the formation of the triangular combustion zone, more chemical energy is released behind the shock wave, thus resulting in higher pressure and temperature in this area. Compared with Fig.5(a), pressure and temperature behind the shock wave in the upper half part in Fig.5(d) have increased $32.56 \%$ and $47.89 \%$, respectively. As a result, as shown in Fig.6(a), a reactive pocket is initially generated in this area behind the shock wave. The reactive pocket grows larger gradually in the region of high pressure and temperature, and then the DDT is induced immediately, as shown in Fig.6(b)(c). This transition finally results in detonation initiation in the upper half part.

Fig. 7 shows the overall structure of the flow field which is fully initiated. In the upper half part is the normal detonation wave tightly coupled with the combustion zone 
following immediately behind. The generated slip line and oblique shock wave on the interface stretch continuously to the lower half part. These form together a typical structure of lateral detonation expansion. The slip line is gradually developed into a shear layer with large-scale vortices because of $\mathrm{KH}$ instabilities, and the angle of the oblique shock wave is about $29.25^{\circ}$. Behind the oblique shock wave the mixture is also combusted, but the reaction zone is not entirely coupled with the oblique shock wave. Near the wall in the lower half part is a short Mach stem which can be shown to be essentially a locally overdriven Mach detonation with a slip line following behind the triple point [44].

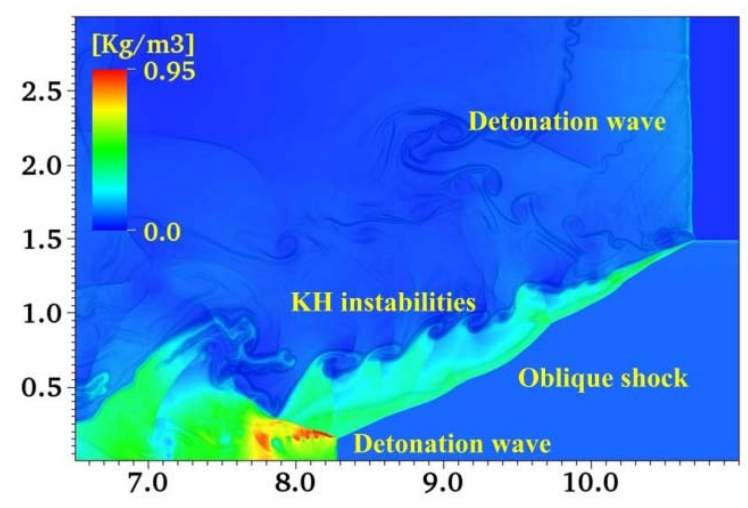

Fig.7 The overall structure after the detonation is fully initiated shown by a density color plot and an overlaid numerical schlieren image of the $\mathbf{O H}$ mass fraction at $t=351.12 \mu \mathrm{s}$.

Fig. 8 shows the location history of detonation front in the upper half part after detonation initiation is finally achieved there at $t=290 \mu \mathrm{s}$. It seems that the curve is initially almost a straight line as denoted as Stage A in Fig.8, which indicates that the detonation propagates at a constant velocity. The propagation velocity equals to about $v=1820 \mathrm{~m} / \mathrm{s}$, which is represented by the slope of the line in Stage A, and then the absolute velocity can be obtained by $V=V_{C J}+v=3447 \mathrm{~m} / \mathrm{s}$. The CJ velocity for 
$\mathrm{M}_{2}$ under this specific condition is $V_{C M_{2}}=2688.7 \mathrm{~m} / \mathrm{s}$, which indicates that the detonation in the upper half part is actually overdriven, with an overdrive degree of $f=1.64\left(f=\left(\frac{V}{V_{C J M_{2}}}\right)^{2}\right)$.

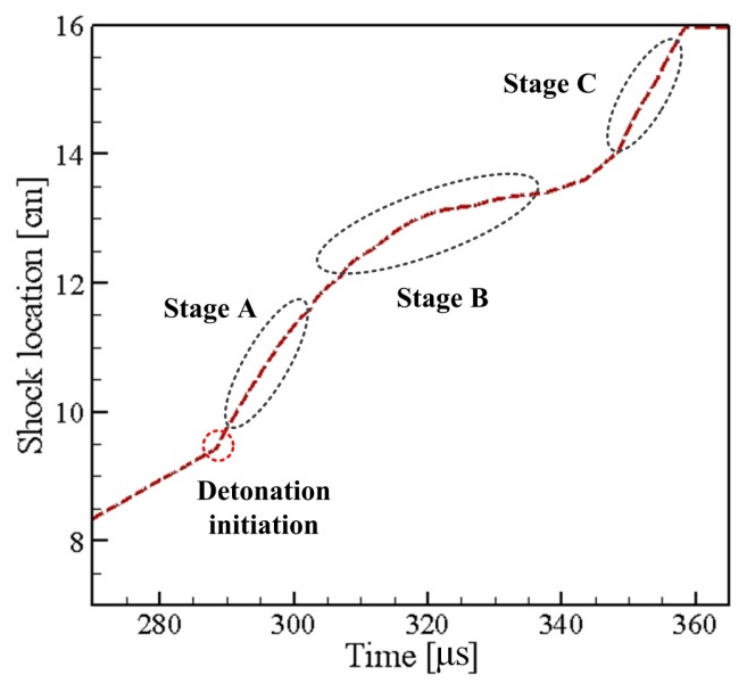

Fig.8 Plot of the location history of the detonation front in the upper half part, which is

\section{divided into three stages.}

As a result, high-pressure products behind the overdriven detonation expand gradually, which finally results in the disappearance of the Mach stem in the lower half part, as shown in Fig.9(a). However, the overdriven detonation cannot be sustained without sufficient energy released from the reaction behind the detonation front. In the lateral expansion zone behind the detonation wave, pressure and temperature decline gradually and subsequently slow down the rate of chemical reaction. Therefore, it is not possible at this point to continuously support the propagation of an overdriven detonation. As shown in Fig.9(b) an attenuation occurs during the propagation in the upper half part in which the reaction front is obviously decoupled from the shock wave. This attenuation results in a decrease of the propagation velocity which corresponds to 
Stage B of Fig.8, and further decreases the pressure and temperature behind the detonation wave. In Fig.9(b), the pressure and temperature behind the shock wave have decreased about $55.8 \%$ and $69.7 \%$, respectively, compared with that in Fig.7. On the other hand, when the pressure in the lateral expansion region decreases, the oblique shock wave gradually lifts up, increases its angle and finally results in the reformation of a new Mach stem and actually a local overdriven Mach detonation, as shown in Fig.9(c). This newly formed local detonation grows stronger and its front Mach stem becomes higher. In this way, products behind the detonation wave cannot expand as freely as before, thus gradually preventing the lateral expansion in the upper half part. As shown in Fig.9(d), the transition on the other hand prompts the increase of pressure and temperature in the lateral expansion zone and induces the reformation of an overdriven detonation in the upper half part, which corresponds to Stage C in Fig.8. The relative propagation velocity in Stage $C$ is approximately $v=1880 \mathrm{~m} / \mathrm{s}$, which is almost the same as that in Stage A, indicating that an entire initiation and re-initiation process has been completed. The periodic exchange between lateral expansion of overdriven detonation in the upper half part and formation of a locally overdriven Mach detonation in the lower half part continuously keeps the two different mixtures fully combusted in the channel. 

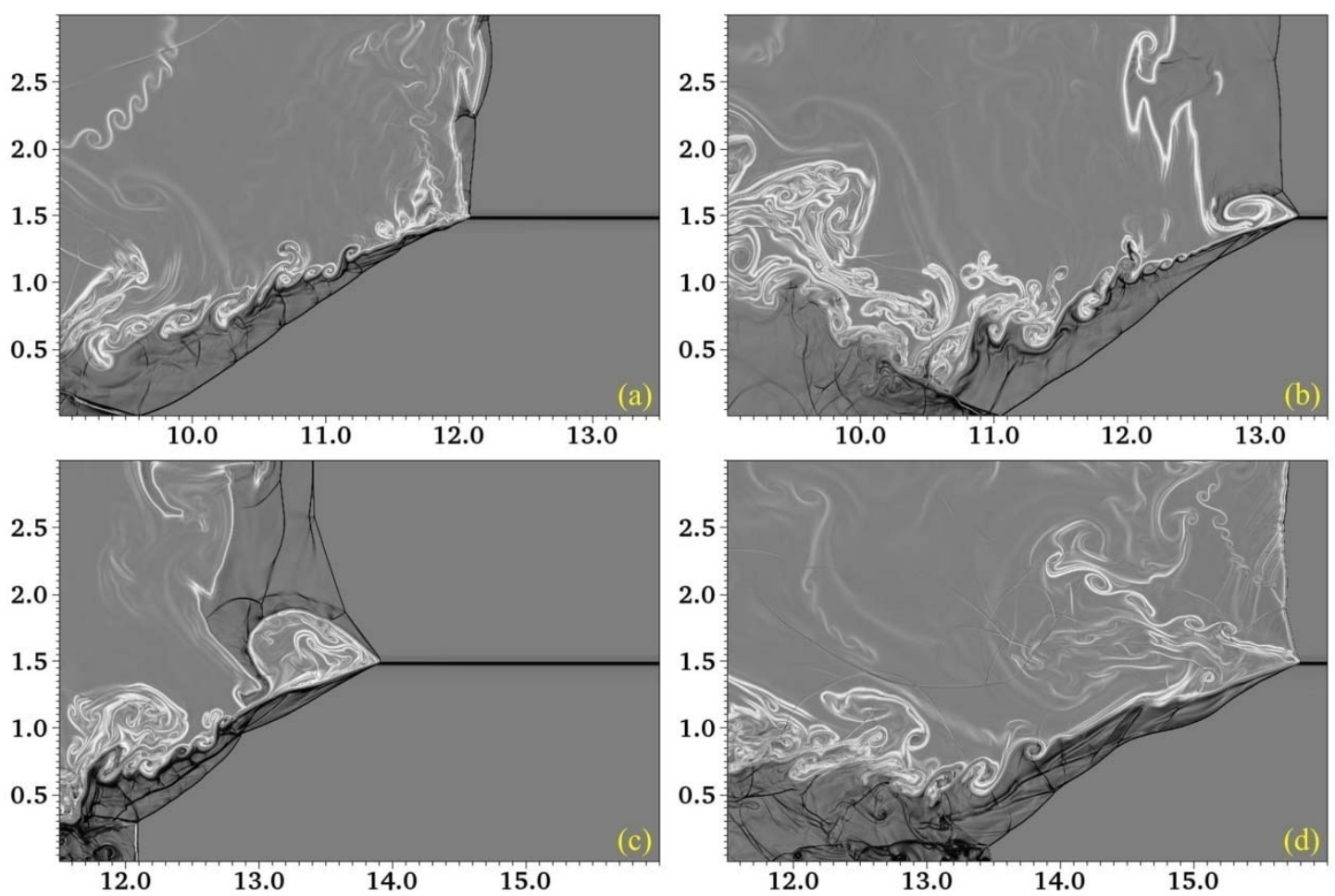

Fig.9 The periodic process after detonation is fully realized in the channel shown by $\mathrm{OH}$

mass fraction schlieren images (white) and density schlieren images (black). (a)

$t=307.06 \mu \mathrm{s}$; (b) $t=322.73 \mu \mathrm{s}$; (c) $t=340.92 \mu \mathrm{s}$; (d) $t=356.78 \mu \mathrm{s}$.

\subsubsection{Mixture Group G1.2}

For $\mathrm{G} 1.2$, the mixtures $\mathrm{M}_{1}$ and $\mathrm{M}_{2}$ are just interchanged compared to G1.1, while the other conditions are kept the same. Fig.10(a) shows the hot jet injection into the channel and formation of the shock reflection on the upper wall. Compared with Fig.3(a), the bow shock here is more abrupt in the lower half part and there is no obvious corner generated on the interface. This is because the strength of the induced bow shock is mainly determined by the momentum flux ratio $J$ $\left(J=\gamma_{j} P_{j} M a_{j}^{2} / \gamma_{\infty} P_{\infty} M a_{\infty}^{2}=\gamma_{j} P_{j} M a_{j}^{2} /\left(P_{\infty} V_{C J}^{2} / R T\right)\right)$ [29], which here is decided only by the characteristic parameter $R$ of $\mathrm{M}_{1}$ in G1.2. The reflective shock wave subsequently reflects again on the vortices resulting from $\mathrm{KH}$ instabilities and forms a triangular reflection zone, as shown in Fig.10(b). Because of the interactions between 
the reflected shock wave and vortices, the flow field around the triangular zone becomes more unstable, and small-scale vortices grow into large-scale ones. The interactions and diffusion effects strengthened by the growing instabilities finally prompt the formation of the Mach stem on the upper wall, as shown in Fig.10(c). Different from the pure Mach stem in Fig.3(b), behind the Mach stem there is a combustion zone, which is believed to be a local detonation wave with combustion zone tightly following behind [29]. The formation of the Mach stem as a local Mach detonation indicates that detonation initiation is successfully realized in the upper half part. However, detonation combustion is still not realized in the lower half part, although a normal shock wave is already generated there.
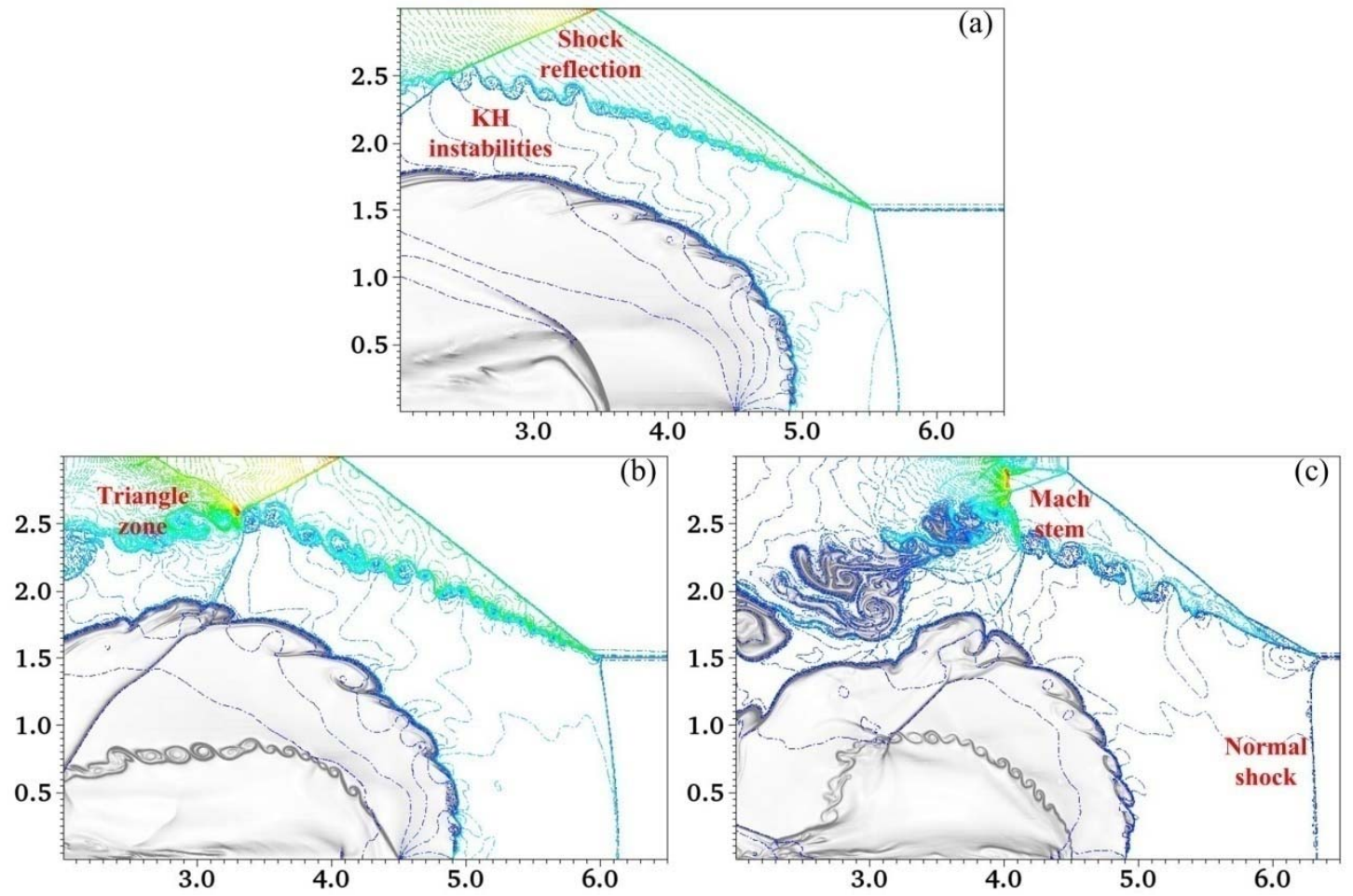

Fig.10 Formation of the Mach stem as a local Mach detonation in the upper half part shown

by density isolines and schlieren images of $\mathrm{OH}$ mass fractions. (a) $t=60.28 \mu \mathrm{s}$; (b)

$$
t=120.71 \mu \mathrm{s} ; \text { (c) } t=167.15 \mu \mathrm{s} \text {. }
$$


The initiation process in the lower half part is shown in detail in Fig.11. At first, deflagration combustion is formed in the upper half part because of the interactions between the reflective shock wave and large-scale vortices, as shown in Fig.11(a). After undergoing a transient process, a detonation bubble is realized abruptly through the DDT mechanism, as shown in Fig.11(b). The localized detonation propagates towards the unreacted mixture which has been already compressed by the shock wave in the front, thus quickly initiating a detonation fully in the lower half part, as shown in Fig.11(c). Detonation initiation in G1.2 is realized more quickly in a total time of $t=190 \mu$ s, while in G1.1 a total time of $t=290 \mu$ s is required.

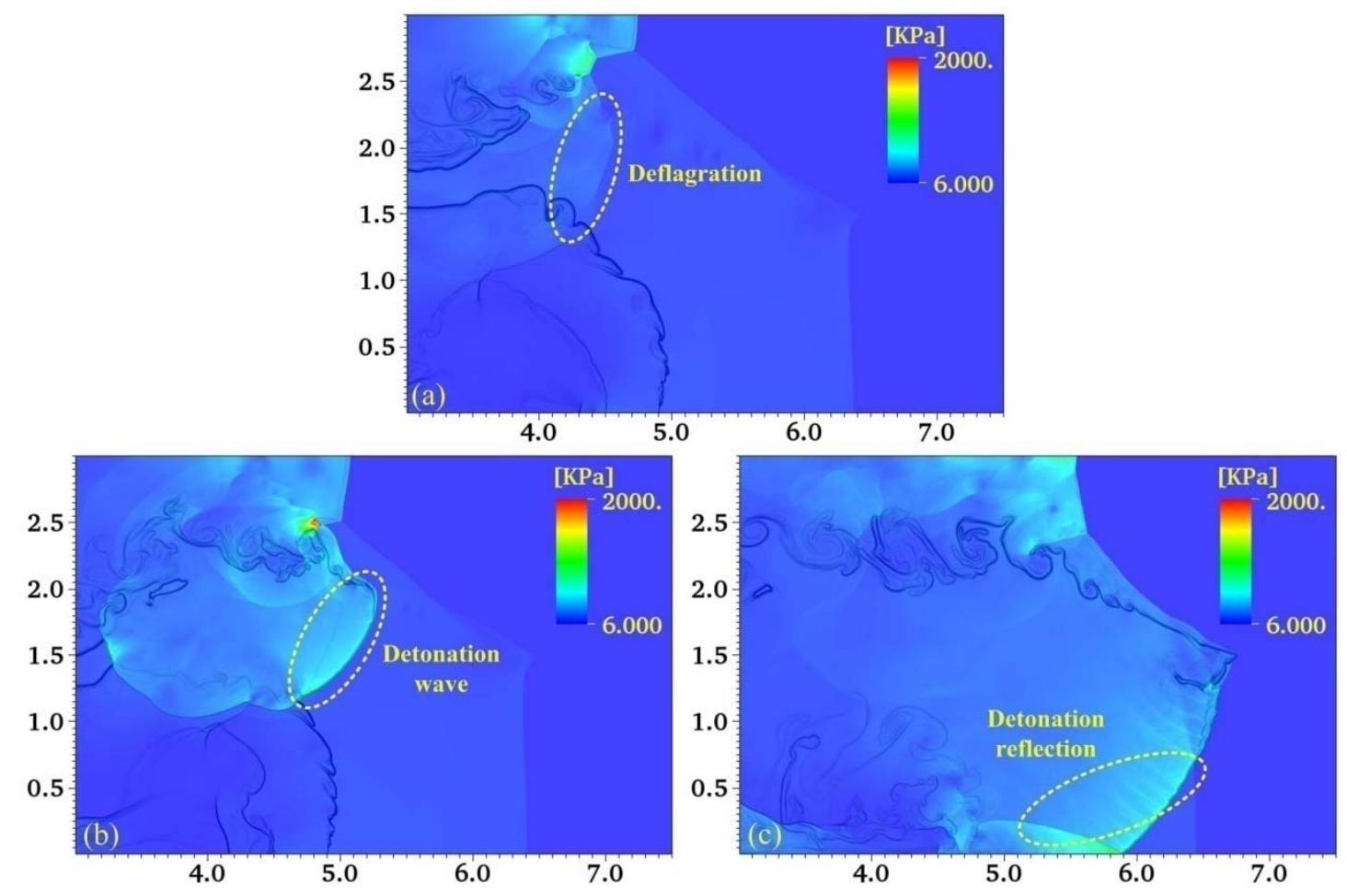

Fig.11 Detonation initiation in the lower half part through DDT mechanism shown by

pressure contours and $\mathrm{OH}$ mass fraction schlieren images. (a) $t=174.35 \mu \mathrm{s}$; (b)

$$
t=181.67 \mu \mathrm{s} ; \text { (c) } t=188.69 \mu \mathrm{s} \text {. }
$$

The curve in Fig.12 has almost the same shape as that in Fig.8, which indicates 
that there also exists a periodical transition for the mixtures G1.2. The overdrive degree of the detonation in the lower half part in Stage A in Fig.12 is approximately $f=1.2$, which is $26.8 \%$ lower than that in Fig. 8 . In supersonic combustible mixtures, the hot jet can play an important role in detonation propagation by preventing the expansion of the products behind the detonation wave through the continuous hot jet injection [27]. When the distribution of two different mixtures is interchanged, the relative position between the hot jet and two different mixtures is also changed, thus resulting in different effects on detonation propagation. In G1.2, the generated local Mach detonation in the upper half part is not as strong as that in G1.1, because the hot jet in the lower half part does not block the expansion of the products behind the Mach stem as largely as that in G1.1. Therefore, a relatively weaker detonation in the upper half part should make a weaker impact on compressing the expansion channel for detonation in the lower half part. As a result, the overdrive degree in G1.2 is relatively lower than that in G1.1.

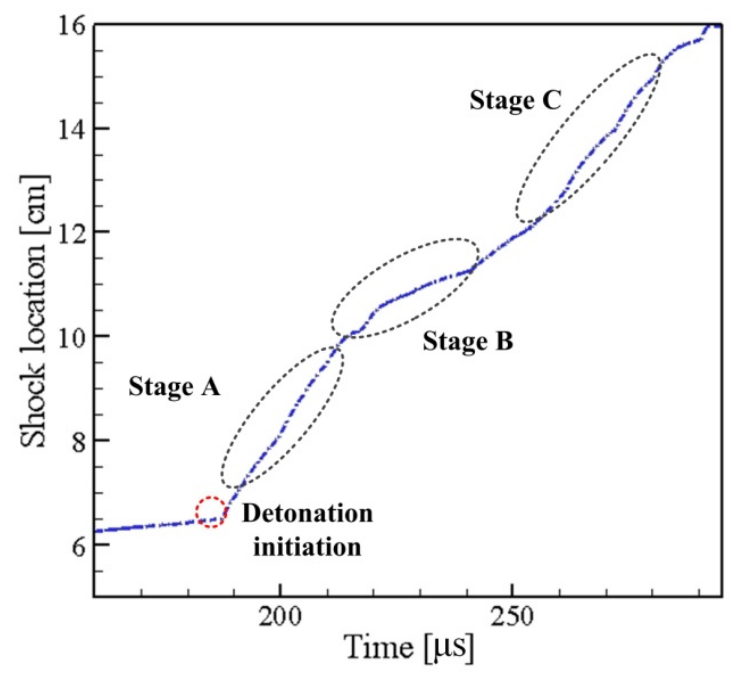

Fig.12 The location history of the detonation front in the lower half part after $t=190 \mu \mathrm{s}$ when detonation initiation is fully realized in the channel. The whole curve is 


\section{divided into three stages.}

Four stages for the whole periodical transition are shown in Fig.13, which can generally be matched to corresponding snapshots of Fig.9. Especially in Fig.13(a) the overall structure of a fully detonated flow field includes both the local overdriven Mach detonation in the upper half part and a lateral expansion of the detonation in the lower half part, which is similar with that in Fig.7. Compared with Fig.9, the difference is that in Fig. 13 the Mach stem in the upper half part never disappears, which is the result of the less overdriven detonation in the lower half part.
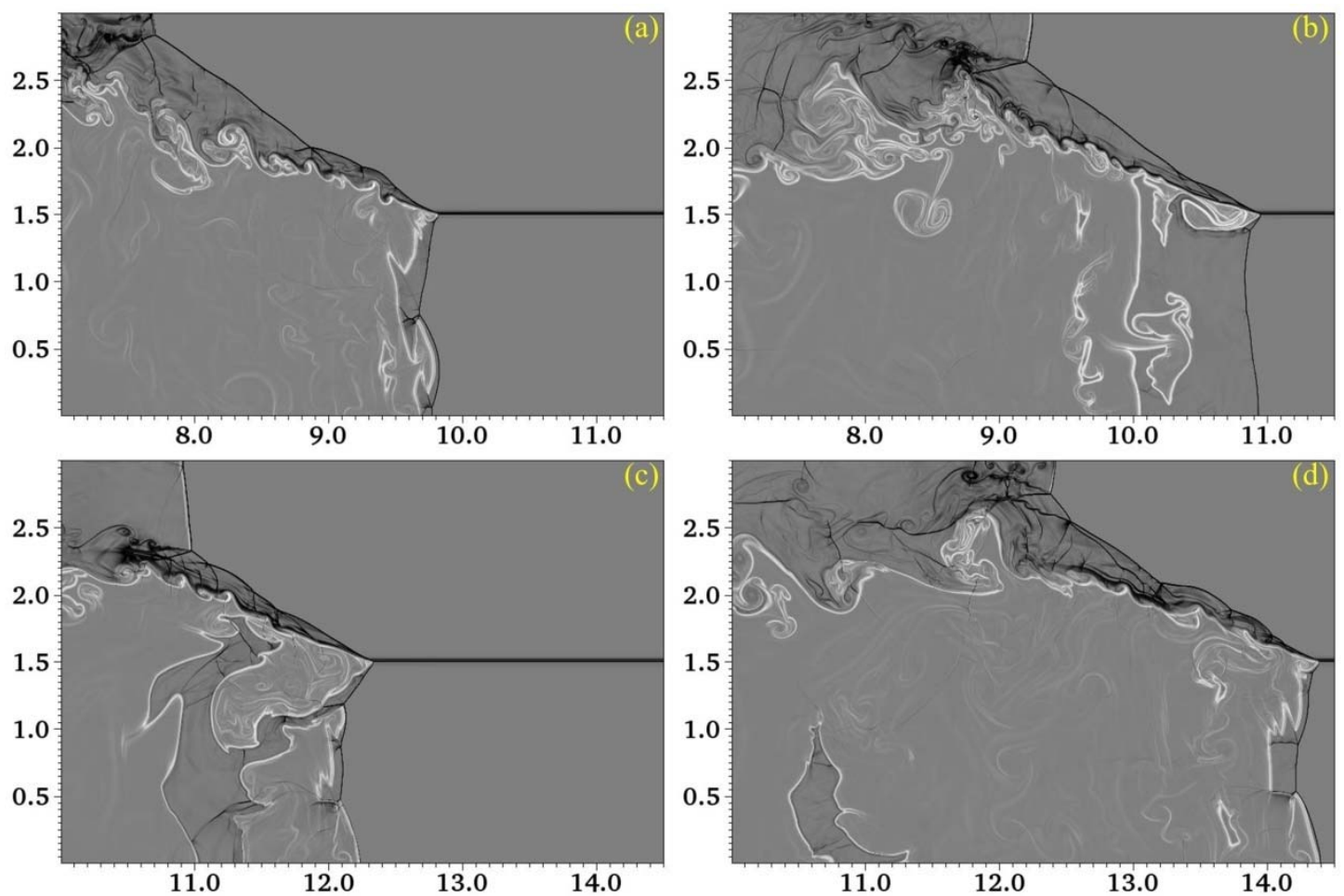

Fig.13 Periodical process after detonation is fully realized in the channel shown by $\mathrm{OH}$ mass

fraction schlieren images (white) and density schlieren images (black). (a) $t=212.07 \mu \mathrm{s}$;

(b) $t=229.96 \mu \mathrm{s}$; (c) $t=256.40 \mu \mathrm{s}$; (d) $t=274.48 \mu \mathrm{s}$.

In group G1, where the mixtures in general have a high degree of chemical reactivity, a detonation can be successfully initiated through Mach reflection and the 
DDT mechanism, independent of the spatial distribution of two the different mixtures.

A recurring four-stage sequence of detonation initiation, detonation attenuation, initiation failure and detonation reinitiation has been identified. This periodic process is also affected by the lateral expansion of the detonation wave. Under lateral expansion, the overdrive degree shows minor variations, which is a result of the hot jet and interchanging the two different mixtures.

\subsection{Results for Mixture Group G2}

\subsubsection{Mixture Group G2.1}

Fig.14 shows the detailed process of detonation initiation for G2.1. After the hot jet is injected into the channel, a bow shock is initially induced, grows gradually stronger and eventually reflects on the upper wall, as shown in Fig.14(a). As the shock reflection is getting stronger, a Mach stem is formed as shown in Fig.14(b), which can be proven to be a locally overdriven Mach detonation [29]. In Fig.14(b), even the second triple point can be distinguished. It obviously followed by a slip line which gradually develops to large-scale vortices because of $\mathrm{KH}$ instabilities. The Mach stem propagates forward along the bow shock continuously and finally reaches the lower wall to generate a second reflection, as shown in Fig.14(c). The second reflection as an ignition source can help realize detonation initiation successfully in the whole channel. With the continuous injection of the hot jet, detonation propagates forward undisturbed as shown in Fig.14(d). 

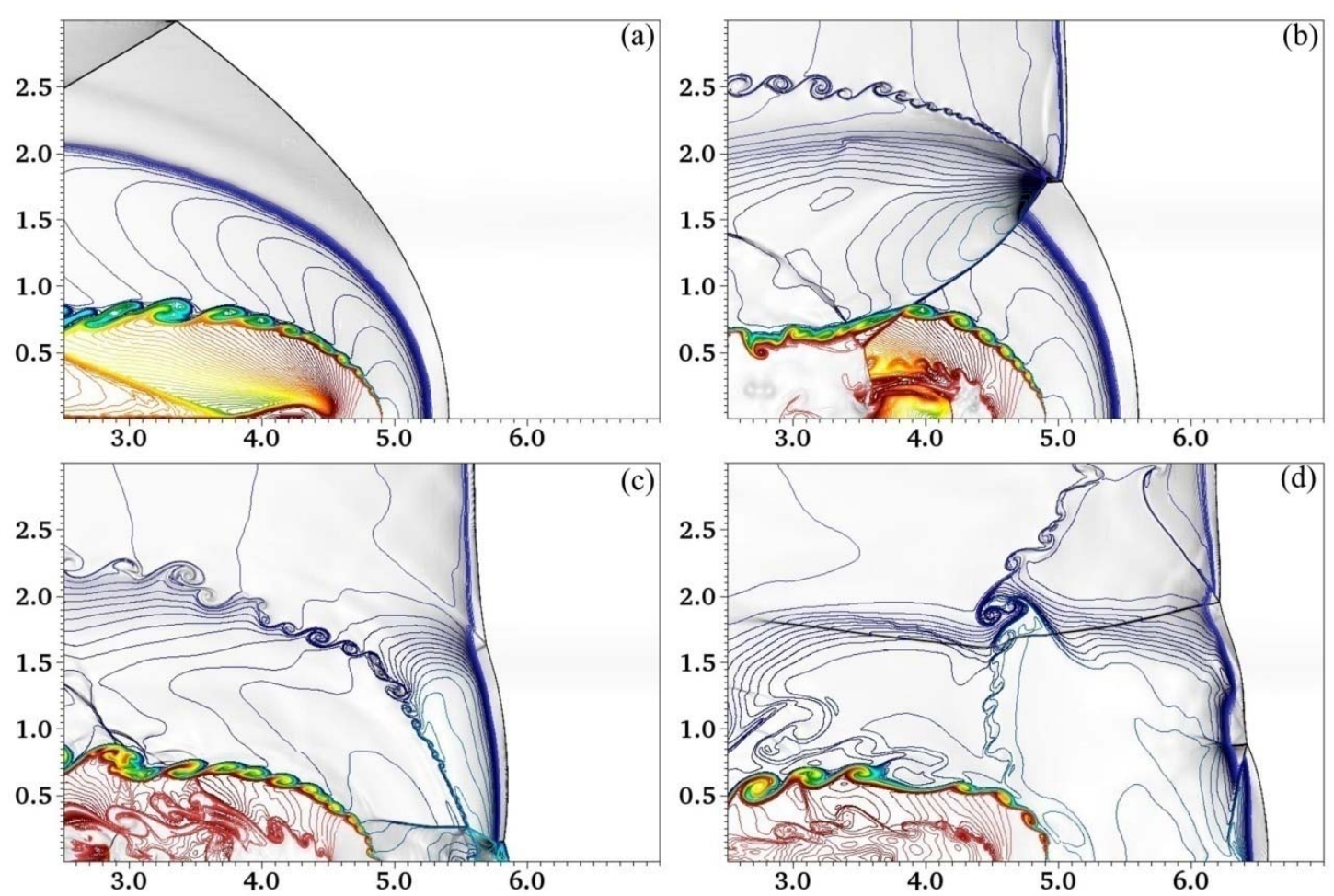

Fig.14 Detonation initiation processes shown by density schlieren images and $\mathrm{OH}$ mass

fraction isolines. (a) $t=84.86 \mu \mathrm{s}$; (b) $t=183.2 \mu \mathrm{s}$; (c) $t=203.78 \mu \mathrm{s}$; (d)

$$
t=238.68 \mu \mathrm{s} .
$$
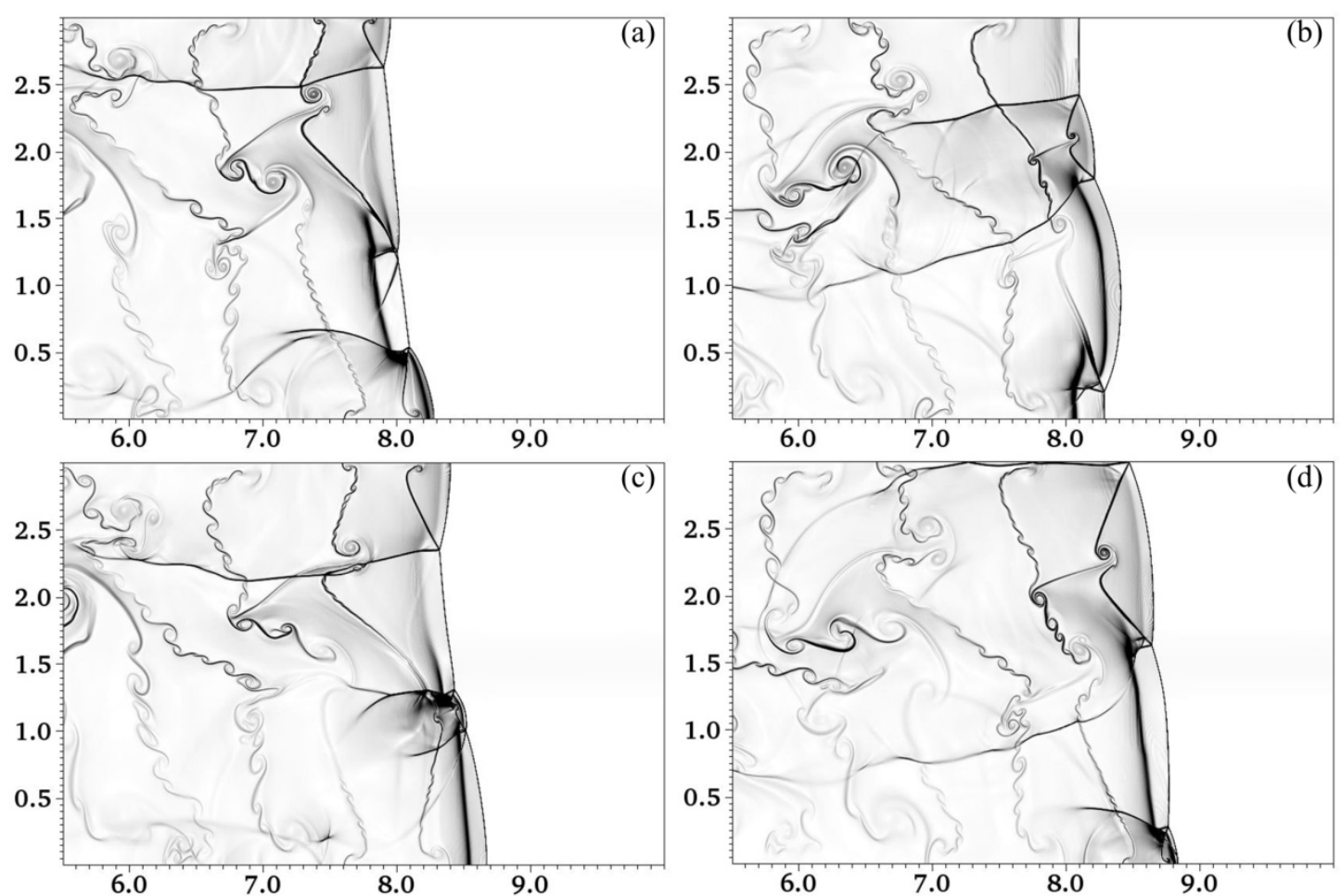

Fig.15 Overdriven detonation propagation with continuous injection of the hot jet. (a) 


$$
t=342.41 \mu \mathrm{s} \text {; (b) } t=355.61 \mu \mathrm{s} \text {; (c) } t=368.58 \mu \mathrm{s} \text {; (d) } t=381.85 \mu \mathrm{s} \text {. }
$$

During the latter period the detonation spreads through the entire channel, as shown in Fig.15. Although mixtures in the channel are divided into two different parts, the detonation fronts almost propagate quite similarly. The CJ velocities for $\mathrm{M}_{1}$ and $\mathrm{M}_{2}$ are $V_{C J M_{1}}=1781.6 \mathrm{~m} / \mathrm{s}$ and $V_{C J M_{2}}=1525.7 \mathrm{~m} / \mathrm{s}$, respectively. Fig.16(a) shows the location history of the overall detonation front after successful initiation. It is suggested that the curve has almost a straight trend. The relative propagation velocity of the whole detonation can be obtained by calculating the line slope which is about $v=165.03 \mathrm{~m} / \mathrm{s}$. The absolute velocity for the whole detonation is $V=v+V_{C J}=1792.03 \mathrm{~m} / \mathrm{s}$. Therefore, overdrive degrees for $\mathrm{M}_{1}$ and $\mathrm{M}_{2}$ are $f_{M_{1}}=1.012$ and $f_{M_{2}}=1.379\left(f=\left(V / V_{C J M}\right)^{2}\right)$, respectively. This indicates that initiated detonations for two different mixtures are both overdriven. Detonation for $\mathrm{M}_{1}$ in the lower half part is only slightly overdriven, while detonation for $\mathrm{M}_{2}$ is strongly overdriven, thus keeping the same traveling pace together with that in the lower half part. The formation of an overdriven detonation is a result of the continuous hot jet injection [27]. Because of the combination of two different mixtures in the channel, the detonation front in Fig.15 varies considerably, which is different from that in uniform supersonic combustible mixtures [65]. However, according to Fig.16(a), oscillations of the detonation front are very regular with an oscillation period of $\Delta T=21.72 \mu \mathrm{s}$. 

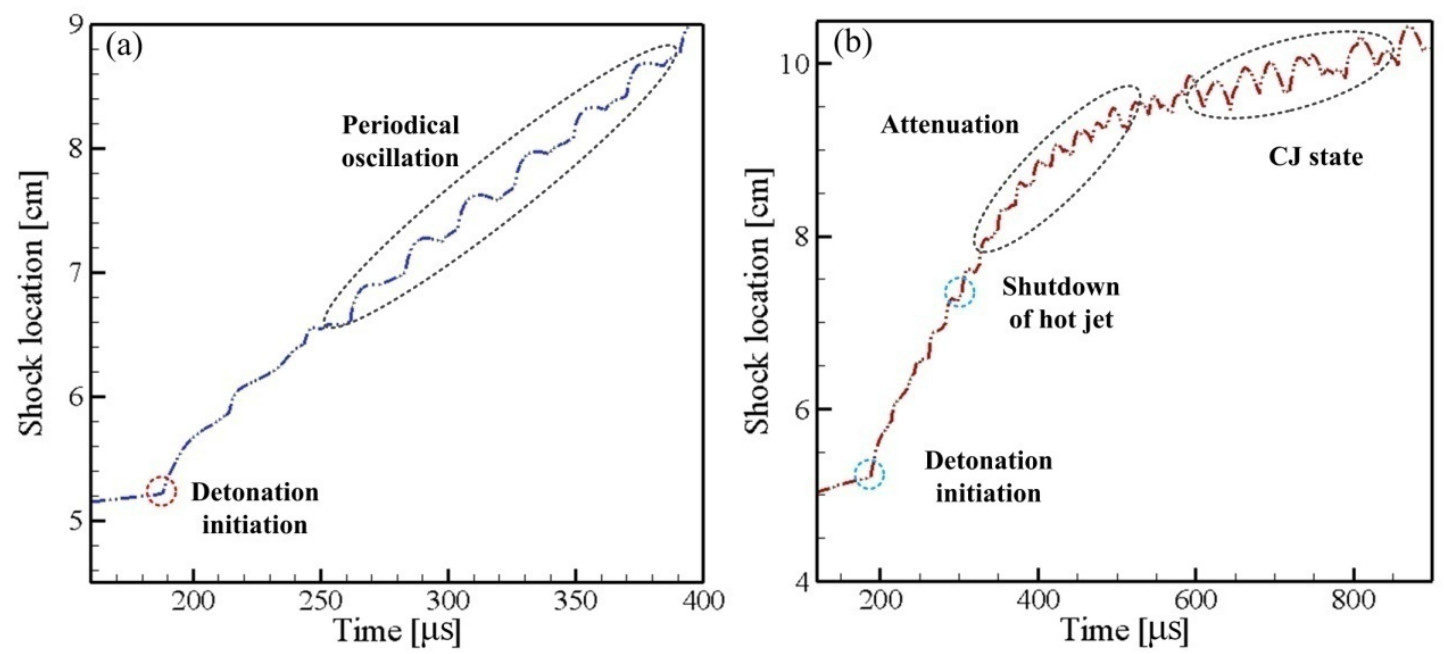

Fig.16 The location history of the shock wave on the mixture interface. (a) with continuous

hot jet injection; (b) after the shutdown of the hot jet at $t=300 \mu \mathrm{s}$.

It is reported that when the hot jet is shut down, the overdriven detonation attenuates and finally reaches a dynamically stable CJ state in a straight channel [27]. Fig.16(b) shows the location history of the overall detonation after the shutdown of the hot jet at $t=300 \mu \mathrm{s}$. During the period from $t=300 \mu \mathrm{s}$ to $t=600 \mu \mathrm{s}$ the slope of the curve decreases gradually, indicating that the propagating velocity of the overdriven detonation is slowing down and an attenuation occurs.
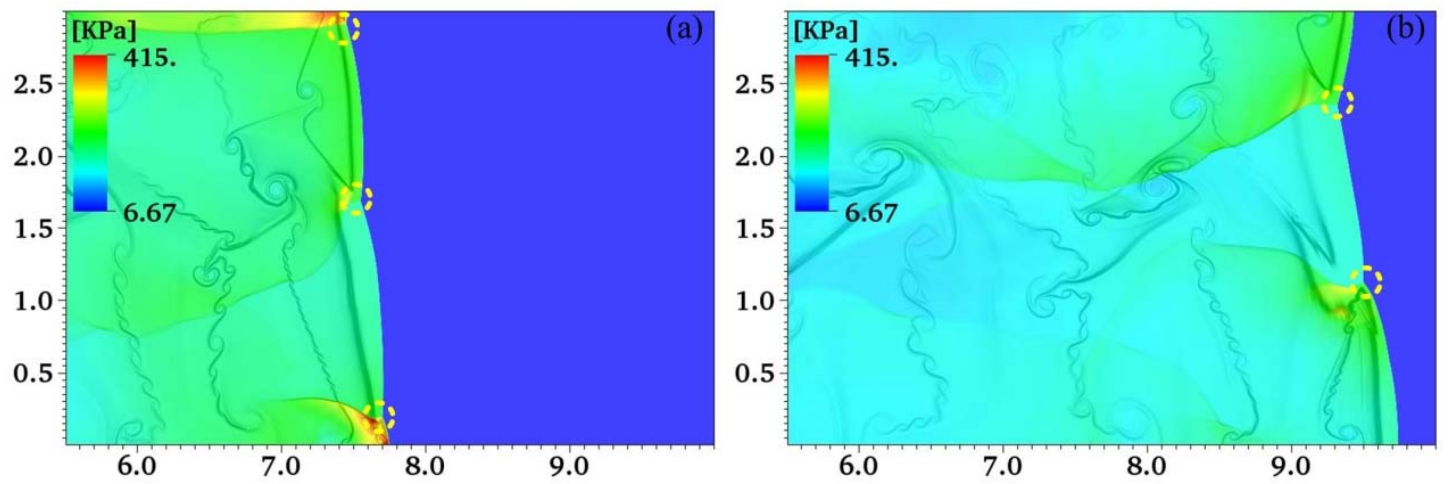

Fig.17 The attenuation of the overdriven detonation shown by pressure color plots overlaid by schlieren images of the $\mathrm{OH}$ mass fractions. (a) $t=316.78 \mu \mathrm{s}$; (b) $t=537.36 \mu \mathrm{s}$.

During the transition from an overdriven to a CJ detonation, transverse waves are weakened gradually and absorbed finally by stronger ones, which can result in a 
reduction of the triple point number, as shown in Fig.17. Three triple points shown by yellow circles in Fig.17(a) are reduced to only two in Fig.17(b). In this way, small detonation cells grow into larger ones, until the formation of the stable CJ state.
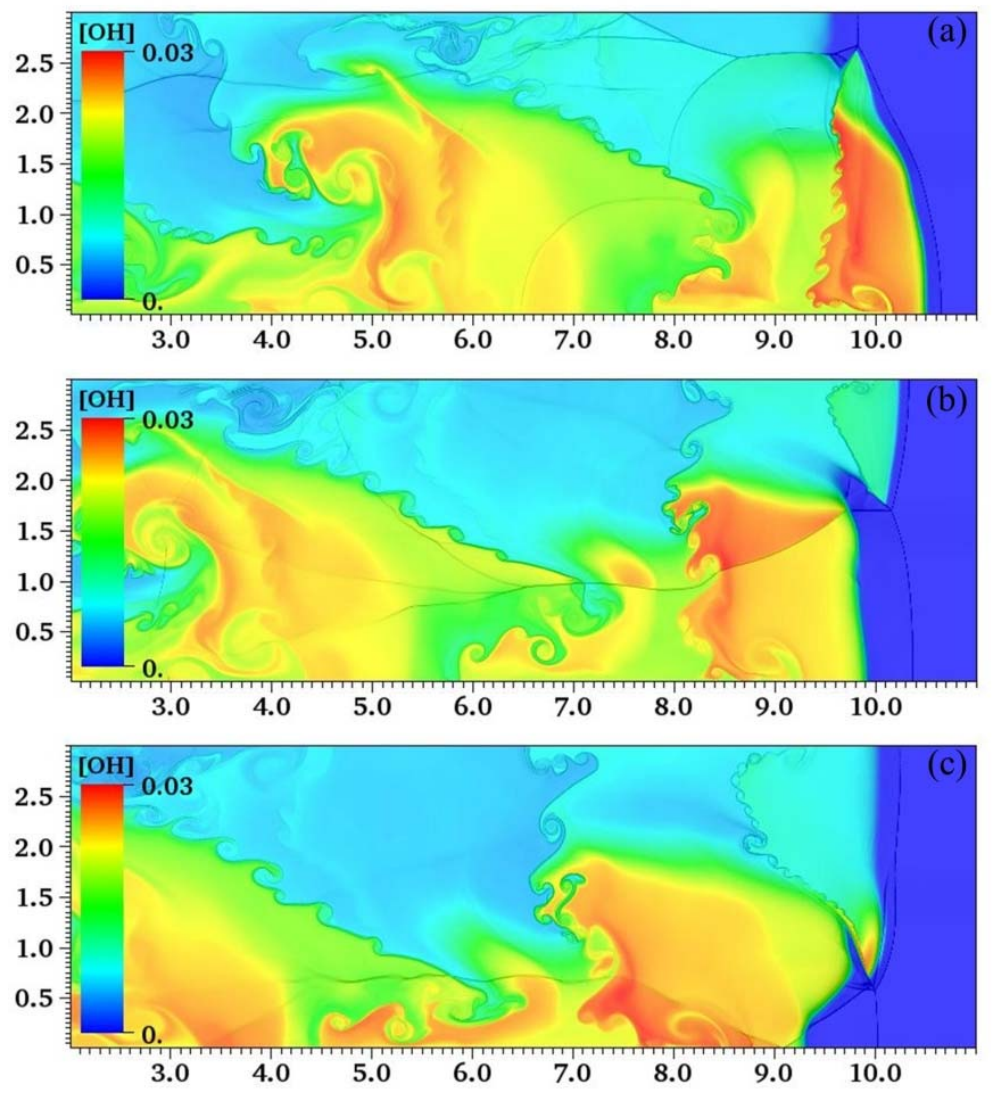

Fig.18 The dynamically stable state of detonation shown by density schlieren images and $\mathrm{OH}$ mass fraction contours. (a) $t=864.64 \mu \mathrm{s}$; (b) $t=885.25 \mu \mathrm{s}$; (c) $t=900 \mu \mathrm{s}$.

Although oscillations are larger, after $t=600 \mu$ s the curve in Fig.16(b) is almost straight, indicating the overall detonation has reached a dynamically stable state. The relative propagation velocity is about $v=23.06 \mathrm{~m} / \mathrm{s}$, which has been obtained by measuring the curve slope. Thus the absolute velocity is obtained as $V=v+V_{C J}=1650.06 \mathrm{~m} / \mathrm{s}$. Therefore, for the detonation in the lower half part with $\mathrm{M}_{1}$ the propagating velocity is $7.38 \%$ lower than the $\mathrm{CJ}$ velocity (underdriven detonation), while for that in the upper half part with $\mathrm{M}_{2}$ the propagating velocity is 
$8.15 \%$ higher than the $\mathrm{CJ}$ velocity (overdriven detonation). Although two different parts of the channel have two different detonation states, their combination presents a new dynamically stable state, as shown in Fig.18. It should be noted that in the eventual stable state, only one triple point is preserved at the detonation front. Because of two different mixtures with different densities, the flow field shows a typical wavy structure in the channel.

\subsubsection{Mixture Group G2.2}

When positions of two different mixtures in G2.1 are interchanged with one another, detonation initiation cannot even be realized. Only a final stable state of the typical structure of shock reflection is formed in the flow field, as shown in Fig.19.

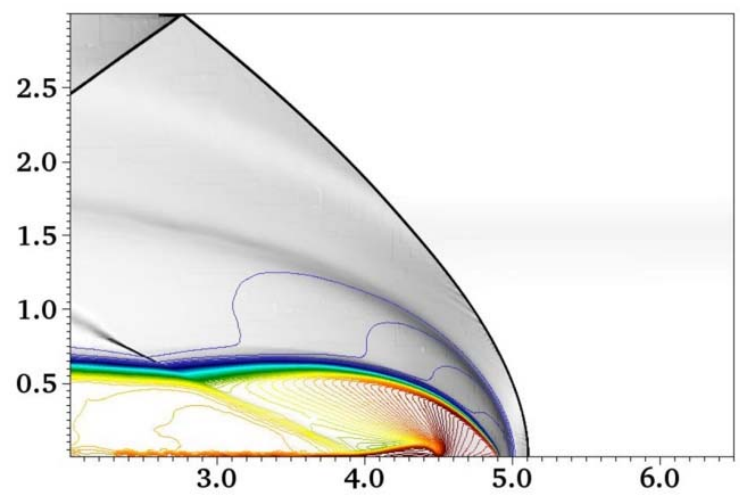

Fig.19 The stable structure of shock reflection on the upper wall

As shown in Section 3.2.2, the strength of the induced bow shock here is only determined by the characteristic parameter $R$ of $\mathrm{M}_{1}$. In $\mathrm{G} 2.2, \mathrm{M}_{1}$ has a relatively small $R$ so that the strength of the bow shock might be lower than the critical value for successful initiation. Compared with G2.1, it is suggested that the mixture $\mathrm{M}_{1}$ in the lower half part, where the hot jet exit is located, might play a more important role in the determination of the bow shock strength than $\mathrm{M}_{2}$ in the upper half part, which is further away from the hot jet exit. 
In group G2, where the mixtures in general have an intermediate degree of chemical reactivity, detonation combustion can be fully realized in the whole channel. After the shutdown of the hot jet, a slightly overdriven detonation and a slightly underdriven detonation are formed together in the flow field. However, it should be noted that in this case whether detonation initiation can be realized or not is mainly determined by the distribution of two different mixtures. In order to address this issue, an effective method is to install the hot jet both on the lower wall and upper wall. In this way, no matter how the mixtures are distributed in the channel, detonation initiation can always be realized successfully.

\subsection{Results for Mixture Group G3}

For G3, detonation initiation cannot be realized successfully neither in G3.1 nor in G3.2 because of the low degree of chemical activity and weak strength of the induced bow shock. Finally, the structure of shock reflection is formed in the flow field, as shown in Fig.20. In group G3, where the mixtures in general have a low degree of chemical activity, the only approach for successful initiation would be the application of stronger hot jets [29], such as increasing the injection pressure, injection velocity, etc. 

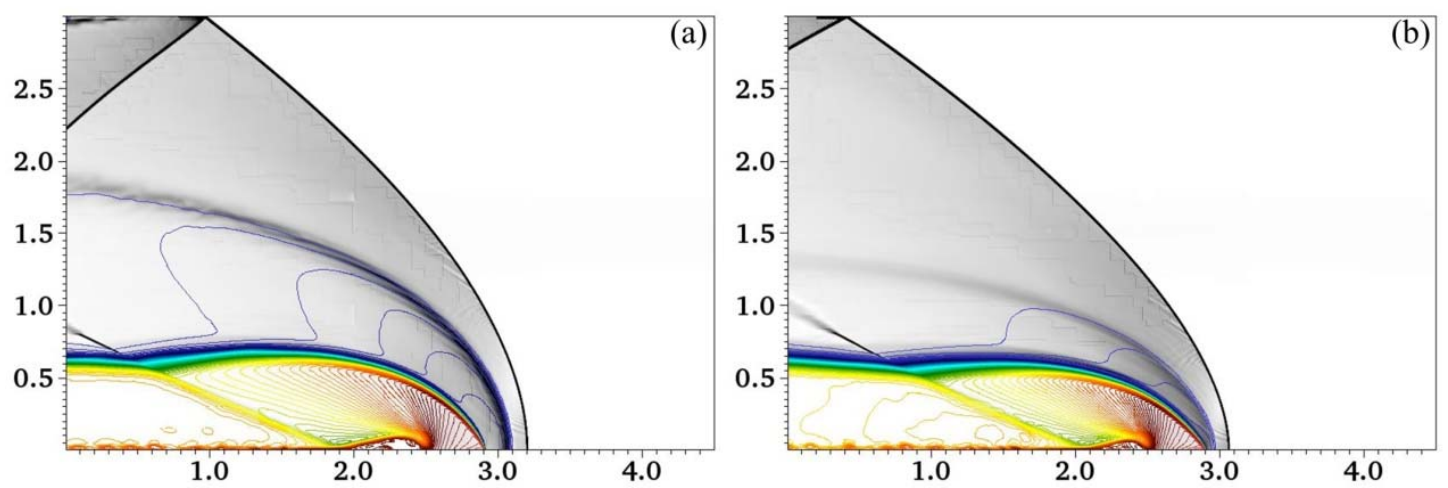

Fig.20 The stable structures of shock reflections on the upper wall.

\section{Discussion}

In supersonic combustible mixtures with non-uniform species, the individual gas constants $(R)$ and the heat capacity ratios $(\gamma)$ are usually different. Although the velocity of supersonic incoming flow is the same, the Mach numbers $(M a=V / \sqrt{\gamma R T})$ in the corresponding mixtures are different. Based on the Rankine-Hugoniot equation $\left(\frac{p-p_{\infty}}{p_{\infty}}=\frac{2 \gamma_{\infty}}{\gamma_{\infty}+1} M a_{\infty}^{2}-\frac{2 \gamma_{\infty}}{\gamma_{\infty}+1}\right)$, the strength of the normal shock (Mach stem) is mainly determined by the Mach number of the incoming flow. In G1.1 for example, the Mach number for $\mathrm{M}_{1}$ in the lower half part is 4.655 while for $\mathrm{M}_{2}$ in the upper half part it is only 3.026. Therefore, the strength of the generated Mach stem for $\mathrm{M}_{1}$ in the lower half part is approximately 2.5 times of that for $\mathrm{M}_{2}$ in the upper half part. Although the degree of chemical reactivity for $\mathrm{M}_{2}$ in the upper half part is higher than that in the lower half part, detonation initiation is still not realized directly through Mach reflection on the upper wall, while in G1.2 detonation initiation can be realized directly through Mach reflection on the upper wall, and subsequently detonation initiation is also induced in the lower half part quickly through the DDT mechanism. 
Different mixtures in the channel have different CJ velocities. In G1, CJ velocities of two different mixtures have a large difference, where the larger CJ velocity is almost 1.65 times the small CJ velocity. Because of the large difference between the two CJ velocities, detonation waves in the two parts of the channel are always clearly separated, which results in the formation of a lateral expansion of the detonation through the periodical process previously described. This periodical process is actually an automatic adaption between two different detonations, which can maintain their continuous propagation and prevent detonation failure while propagating. In G2, the difference between two different $\mathrm{CJ}$ velocities of the two different mixtures is relatively small as the larger CJ velocity is only 1.17 times of the small one. Therefore, two detonation waves can adapt to each other during propagation, thus resulting in one slightly overdriven detonation and another slightly underdriven detonation. Based on the automatic adaption they eventually form a special steady state together when the final detonation wave for the two different mixtures propagate together at the same velocity.

In G1 and G2, where detonation initiation can be finally realized, detonations in different parts of the channel can adapt automatically to each other, independent of the lateral expansion of the detonation in G1 or the special stable state of the detonation in G2. Because of the automatic adaption in non-uniform species, the initiated detonation can maintain its propagation continuously as a whole, although there may exist local detonation failures partly in the flow field. Based on the lateral expansion of the detonation, it is suggested that the automatic adaption of detonations between different 
mixtures can be adjusted through the distribution of the mixture composition. The lateral expansion of the detonation is significantly influenced by the relative height of the two different mixtures. Different distribution of the two mixtures can play an important role in the formation and evolution of the periodical lateral detonation. Even for the special stable state of the detonation in G2, adjustment of the mixture distribution also has an influence on the development of the eventually typical wavy structure. If the mixtures are not equally distributed through the change of mixture composition in the channel with a fixed height or the height change of the channel, the detonation is thought to be slightly different. Nevertheless, it is suggested that the detonation may maintain its propagation continuously through an automatic adaption mechanism, which is in need of future investigations.

\section{Conclusions}

Based on various degrees of chemical reactivity, detonation initiation and propagation in supersonic combustible mixtures with a spatially non-uniform distribution of two different mixtures was investigated through two-dimensional simulations adopting the open-source program AMROC, and the mechanism was analyzed in detail.

When the mixtures in general have a high degree of chemical reactivity, detonation initiation can be finally realized successfully both through Mach reflection and the DDT mechanism in the flow field, independent of the mixture distribution throughout the channel. In the flow field, four processes of detonation initiation, detonation attenuation, 
initiation failure and detonation reinitiation have been identified. Their successve occurrence creates a periodic transition process in interaction with lateral detonation expansion. It is believed that this periodic process plays an important role in maintaining the continuous detonation in the channel.

When the mixtures in general have a medium degree of chemical reactivity, a detonation can be fully realized in the whole channel with different overdrive degrees in the upper half and the lower half part. When the hot jet is shut down, the overdriven detonation attenuates gradually, and finally a slightly overdriven detonation and a slightly underdriven detonation are formed together, which can be regarded as a new stable state of detonation. However, it should be noted that whether detonation initiation can be realized or not in this case is determined by the distribution of different mixtures. An effective method for addressing this problem is to install the hot jet on both the lower and the upper wall. In this way, no matter how the mixtures are distributed in the channel, detonation initiation can always be realized successfully.

When the mixtures have a low degree of chemical reactivity, detonation initiation cannot be realized. The reliable approach for successful detonation initiation in this case should be applications of stronger hot jets.

\section{Acknowledgements}

This work is supported by National Natural Science Foundation of China (No. 91016028), Innovative Sustentation Fund for Excellent Ph.D. Students in NUDT (No. B140101) and Chinese Scholarship Council (CSC) (No. 201403170401). 


\section{References}

[1] Murthy, S.N.B., and Curran, E.T., "High-Speed Flight Propulsion Systems," Progress in Astronautics and Aeronautics, Vol. 137, 1991, pp. 124-158.

[2] Doherty, L.J., Smart, M.K., and Mee, D.J., "Experimental Testing of an Airframe-Integrated Three-Dimensional Scramjet at Mach 10,” AIAA Journal, Vol. 53, No. 11, 2015, pp. 3196-3207. doi:10.2514/1.J053785

[3] Barth J.E., Wheatley V., and Smart M.K., "Effects of Hydrogen Fuel Injection in a Mach 12 Scramjet Inlet,” AIAA Journal, Vol. 53, No. 10, 2015, pp. 2907-2919.

doi:10.2514/1.J053819

[4] Kailasanath, K., "Review of Propulsion Applications of Detonation Waves," AIAA Journal, Vol. 38, No. 9, 2000, pp. 1698-1708.

doi: $10.2514 / 2.1156$

[5] Zhang, B., Kamenskihs, V., Ng, H.D., and Lee, J.H.S., "Direct Blast Initiation of Spherical Gaseous Detonations in Highly Argon Diluted Mixtures," Proceedings of the Combustion Institute, Vol. 33, No. 2, 2011, pp. 2265-2271.

doi:10.1016/j.proci.2010.06.165

[6] Zhang, B., Ng, H.D., Mével, R., and Lee, J.H.S., "Critical Energy for Direct Initiation of Spherical Detonations in $\mathrm{H}_{2} / \mathrm{N}_{2} \mathrm{O} / \mathrm{Ar}$ Mixtures," International Journal of Hydrogen Energy, Vol. 36, No. 9, 2011, pp. 5707-5716.

doi:10.1016/j.ijhydene.2011.01.175

[7] Zhang, B., Ng, H.D., and Lee, J.H.S., "Measurement of Effective Blast Energy for Direct Initiation of Spherical Gaseous Detonations from High-Voltage Spark Discharge," Shock 
Waves, Vol. 22, No. 1, 2012, pp. 1-7.

doi: 10.1007/s00193-011-0342-y

[8] Knystautas, R., Lee, J.H.S., Moen, I.O., and Wagner, H.GG., "Direct Initiation of Spherical Detonation by a Hot Turbulent Gas Jet," Seventeenth Symposium (International) on Combustion, Vol. 17, No. 1, 1979, pp. 1235-1245.

doi:10.1016/S0082-0784(79)80117-4

[9] Moen, I.O., Bjerketvedt, D., Jenssen, A., and Thibault, P.A., "Transition to Detonation in Large Fuel-Air Cloud," Combustion and Flame, Vol. 61, No. 3, 1985, pp. 285-294. doi:10.1016/0010-2180(85)90109-9

[10] Carnasciali, F., Lee, J.H.S., Knystautas, R., and Fineschi, F., "Turbulent Jet Initiation of Detonation," Combustion and Flame, Vol. 84, No. 1-2, 1991, pp. 170-180. doi:10.1016/0010-2180(91)90046-E

[11] Dorofeev, S.B., Bezmelnitsin, A.V., Sidorov, V.P., Yankin J.G., and Matsukov, I.D., "Turbulent Jet Initiation of Detonation in Hydrogen-Air Mixtures," Shock Waves, Vol. 6, No. 2, 1996, pp. 73-78.

doi:10.1007/BF02515190

[12] Lieberman, D.H., Parkin, K.L., and Shepherd, J.E., "Detonation Initiation by a Hot Turbulent Jet for Use in Pulse Detonation Engines," 38th AIAA/ASME/SAE/ASEE Joint Propulsion Conference \& Exhibit, AIAA Paper 2002-3909, 2002.

doi: $10.2514 / 6.2002-3909$

[13] Medvedev, S.P., Khomik, S.V., Olivier, H., Polenov, A.N., Bartenev, A.M., and Gelfand, B.E., "Hydrogen Detonation and Fast Deflagration Triggered by a Turbulent Jet of Combustion 
Products," Shock Waves, Vol. 14, No. 3, 2005, pp. 193-203.

doi:10.1007/s00193-005-0264-7

[14] Hoke, J.L., Bradley, R.P., Gallia, J.R., and Schauer, F.R., "The Impact of Detonation Initiation Techniques on Thrust in a Pulsed Detonation Engine", 44th AIAA Aerospace Sciences Meeting and Exhibit, AIAA Paper 2006-1023, 2006.

doi:10.2514/6.2006-1023

[15] Liu, S.J., Lin, Z.Y., Liu, W.D., Lin, W., and Zhuang, F.C., "Experimental Realization of $\mathrm{H}_{2} / \mathrm{Air}$ Continuous Rotating Detonation in a Cylindrical Combustor," Combustion Science and Technology, Vol. 184, No. 9, 2012, pp. 1302-1317.

doi:10.1080/00102202.2012.682669

[16] Iglesias, I., Vera, M., Sánchez, A.L., and Liñán, A., "Numerical Analyses of Deflagration Initiation by a Hot Jet,” Combustion Theory and Modelling, Vol.16, No. 6, 2012, pp. 994-1010. doi:10.1080/13647830.2012.690048

[17] Cai, X.D., Liang, J.H., Lin, Z.Y., and Zhuang, F.C., "Influence Analysis of Geometrical Parameters of Detonation Initiation with a Hot Jet by Adaptive Mesh Refinement Method," 49th AIAA/ASME/SAE/ASEE Joint Propulsion Conference, AIAA Paper 2013-4167, 2013. doi:10.2514/6.2013-4167

[18] Ishii, K., Kataoka, H., and Kojima, T., "Initiation and Propagation of Detonation Waves in Combustible High Speed Flows," Proceedings of the Combustion Institute, Vol. 32, No. 2, 2009, pp. 2323-2330. doi:10.1016/j.proci.2008.05.029

[19] Han, X., Zhou, J., and Lin, Z.Y., "Experimental Investigations of Detonation Initiation by Hot 
Jets in Supersonic Premixed Flows," Chinese Physics B., Vol. 21, No. 12, 2012, 124702.

doi:10.1088/1674-1056/21/12/124702

[20] Han, X., Zhou, J., Lin, Z.Y., and Liu, Y., "Deflagration-to-Detonation Transition Induced by Hot Jets in a Supersonic Premixed Airstream,” Chinese Physics Letters, Vol. 30, No. 5, 2013, 054701.

doi:10.1088/0256-307X/30/5/054701

[21] Gamezo, V.N., Ogawa, T., and Oran, E.S., "Flame Acceleration and DDT in Channels with Obstacles: Effect of Obstacle Spacing," Combustion and Flame, Vol. 155, No. 1-2, 2008, pp. 302-315.

doi:10.1016/j.combustflame.2008.06.004

[22] Jackson, S.I., and Shepherd, J.E., "Detonation Initiation in a Tube via Imploding Toroidal Shock Waves," AIAA Journal, Vol. 46, No. 9, 2008, pp. 2357-2367. doi:10.2514/1.35569

[23] Melguizo-Gavilanes, J., and Bauwens, L., "Shock Initiated Ignition for Hydrogen Mixtures of Different Concentrations," International Journal of Hydrogen Energy, Vol. 38, No. 19, 2013, pp. 8061-8067. doi:10.1016/j.ijhydene.2013.03.018

[24] Melguizo-Gavilanes, J., Rezaeyan, N., Tian, M., and Bauwens, L., "Shock-Induced Ignition with Single Step Arrhenius Kinetics,” International Journal of Hydrogen Energy, Vol. 36, No. 3, 2011, pp. 2374-2380. doi:10.1016/j.ijhydene.2010.04.138

[25] Driscoll, R., Stoddard, W., George, A.S., and Gutmark, E.J, "Shock Transfer and Shock- 
Initiated Detonation in a Dual Pulse Detonation Engine/Crossover System,” AIAA Journal, Vol. 53, No. 1, 2015, pp. 132-139.

doi:10.2514/1.J053027

[26] Driscoll, R., George, A.S., Stoddard, W., Munday, D., and Gutmark, E.J., "Characterization of Shock Wave Transfer in a Pulse Detonation Engine-Crossover System”, AIAA Journal, Vol. 53, No. 12, 2015, pp. 3674-3685.

doi:10.2514/1.J054045

[27] Cai, X.D., Liang, J.H., Lin, Z.Y., Deiterding, R., Qin, H., and Han, X., “Adaptive Mesh Refinement Based Numerical Simulation of Detonation Initiation in Supersonic Combustible Mixtures Using a Hot Jet,” ASCE Journal of Aerospace Engineering, Vol. 28, No. 1, 2015, 04014046.

doi:10.1061/(ASCE)AS.1943-5525.0000376

[28] Liang, J.H., Cai, X.D., Lin, Z.Y., and Deiterding, R., "Effects of a Hot Jet on Detonation Initiation and Propagation in Supersonic Combustible Mixtures," Acta Astronautica, Vol. 105, No. 1, 2014, pp. 265-277.

doi:10.1016/j.actaastro.2014.08.019

[29] Cai, X.D., Liang J.H., Lin, Z.Y., Deiterding, R., and Liu, Y., "Parametric Study of Detonation Initiation Using a Hot Jet in Supersonic Combustible Mixtures," Aerospace Science and Technology, Vol. 39, 2014, pp. 442-455.

doi:10.1016/j.ast.2014.05.008

[30] Berger, M., "Adaptive Mesh Refinement for Hyperbolic Differential Equations," Ph.D. Dissertation, Stanford University, Stanford, 1982. 
[31] Berger, M., and Oliger, J., "Adaptive Mesh Refinement for Hyperbolic Partial Differential Equations," Journal of Computational Physics, Vol. 53, No. 3, 1984, pp. 484-512. doi:10.1016/0021-9991(84)90073-1

[32] Deiterding, R., "Parallel Adaptive Simulation of Multi-Dimensional Detonation Structures," Ph.D. Dissertation, Brandenburgische Technische Universität Cottbus, Cottbus, 2003.

[33] Liang, Z., Browne, S., Deiterding, R., and Shepherd, J.E., "Detonation Front Structure and the Competition for Radicals," Proceedings of the Combustion Institute, Vol. 31, No. 2, 2007, pp. 2445-2453. doi:10.1016/j.proci.2006.07.244

[34] Deiterding., R., "A Parallel Adaptive Method for Simulating Shock-Induced Combustion with Detailed Chemical Kinetics in Complex Domains," Computers and Structures, Vol. 87, No. 11-12, 2009, pp. 769-783. doi:10.1016/j.compstruc.2008.11.007

[35] Deiterding, R., "High-Resolution Numerical Simulation and Analysis of Mach Reflection Structures in Detonation Waves in Low-Pressure $\mathrm{H}_{2}-\mathrm{O}_{2}$-Ar Mixtures: A Summary of Results Obtained with the Adaptive Mesh Refinement Framework AMROC," Journal of Combustion, Vol. 2011, 2011. doi:10.1155/2011/738969

[36] Ziegler, J.L., Deiterding, R., Shepherd, J.E., and Pullin, D.I., “An Adaptive High-Order Hybrid Scheme for Compressive, Viscous Flows with Detailed Chemistry," Journal of Computational Physics, Vol. 230, No. 20, 2011, pp. 7598-7630. doi:10.1016/j.jcp.2011.06.016 
[37] Westbrook, C.K., " $\mathrm{H}_{2}-\mathrm{O}_{2}-\mathrm{AR}$ Reaction Mechanism from: Chemical Kinetics of Hydrocarbon Oxidation in Gaseous Detonations," Combustion and Flame, Vol. 46, 1982, pp. 191-210. doi:10.1016/0010-2180(82)90015-3

[38] Thomas, G.O., Sutton, P., and Edwards, D.H., "The Behaviour of Detonation Waves at Concentration Gradients," Combustion and flame, Vol. 84, No. 3-4, 1991, pp. 312-322. doi:10.1016/0010-2180(91)90008-Y

[39] Kuznetsov, M.S., Alkseev, V.I., Dorofeev, S.B., Matsukov, I.D., and Boccio, J.L., "Detonation Propagation, Decay, and Reinitiation in Nonuniform Gaseous Mixtures," Proceedings of the Combustion Institute, Vol. 27, No. 2, 1998, pp. 2241-2247. doi:10.1016/S0082-0784(98)80073-8

[40] Sochet, I., Lamy, T., Brossard, J., "Experimental Investigation on the Detonability of Nonuniform Mixtures," Shock Waves, Vol. 10, No. 5, 2000, pp. 363-376. doi:10.1007/s001930000066

[41] Ishii, K., and Kojima, M., "Behavior of Detonation Propagation in Mixtures with Concentration Gradients," Shock Waves, Vol. 17, No. 1-2, 2007, pp. 95-102. doi:10.1007/s00193-007-0093-y

[42] Weber, H.J., Mack, A., and Roth, P., "Combustion and Pressure Wave Interaction in Enclosed Mixtures Initiated by Temperature Nonuniformities," Combustion and Flame, Vol. 97, No. 34, 1994, pp. 281-295. doi:10.1016/0010-2180(94)90021-3

[43] Kim, Y.M., Kim, S.J., Chen, Z.J., and Chen, C.P., "Numerical Simulation of Combustion Wave Propagation in an Air-Fuel Spray Mixture with Temperature Nonuniformity," Numerical Heat 
Transfer Part A: Applications, Vol. 34, No. 1, 1998, pp. 23-41.

doi:10.1080/10407789808913975

[44] Cai, X.D., Liang, J.H., Lin, Z.Y., Deiterding, R., and Zhuang, F.C., "Detonation Initiation and Propagation in Nonuniform Supersonic Combustible Mixtures," Combustion Science and Technology, Vol. 187, No. 4, 2015, pp. 525-536.

doi:10.1080/00102202.2014.958223

[45] Mahmoudi, Y., and Mazaheri, K., "High Resolution Numerical Simulation of the Structure of 2-D Gaseous Detonations," Proceedings of the Combustion Institute, Vol. 33, No. 2, 2011, pp. 2187-2194.

doi:10.1016/j.proci.2010.07.083

[46] Radulescu, M.I., and Lee, J.H.S., "The Failure Mechanism of Gaseous Detonations: Experiments in Porous Wall Tubes," Combustion and Flame, Vol. 131, No. 1-2, 2002, pp. 2946. doi:10.1016/S0010-2180(02)00390-5

[47] Radulescu, M.I., Sharpe, G.J., Lee, J.H.S., Kiyanda, C.B., Higgins, A.J., and Hanson, R.K., "The Ignition Mechanism in Irregular Structure Gaseous Detonations," Proceedings of the Combustion Institute, Vol. 30, No. 20, 2005, pp. 1859-1867.

doi:10.1016/j.proci.2004.08.047

[48] Shepherd, J.E., "Detonation in Gases," Proceedings of the Combustion Institute, Vol. 32, No. 1, 2009, pp. 83-98. doi:10.1016/j.proci.2008.08.006

[49] Radulescu, M.I., Sharpe, G.J., Law, C.K., and Lee, J.H.S., "The Hydrodynamic Structure of 
Unstable Cellular Detonations,” Journal of Fluid Mechanics, Vol. 580, 2007, pp. 31-81. doi:10.1017/S0022112007005046

[50] Gamezo, V.N., Desbordes, D., and Oran, E.S., "Formation and Evolution of Two-Dimensional Cellular Detonations," Combustion and Flame, Vol. 116, No. 1-2, 1999, pp 154-165. doi:10.1016/S0010-2180(98)00031-5

[51] Sharpe, G.J., and Falle, S.A.E., "Two-Dimensional Numerical Simulations of Idealized Detonations," Proceedings of the Royal Society London A, Vol. 456, 2000, pp. 2081-2100. doi:10.1098/rspa.2000.0603

[52] Mach, P., and Radulescu, M.I., "Mach Reflection Bifurcations as a Mechanism of Cell Multiplication in Gaseous Detonations," Proceedings of the Combustion Institute, Vol. 33, No. 2, 2010, pp. 2279-2285. doi:10.1016/j.proci.2010.06.145

[53] Mahmoudi, Y., and Mazaheri, K., "Triple Point Collision and Hot Spots in Detonations with Regular Structure," Combustion Science and Technology, Vol. 184, No. 7-8, 2012, pp. 11351151.

doi:10.1080/00102202.2012.664004

[54] Hu, X.Y., Khoo, B.C., Zhang, D.L., and Jiang, Z.L., "The Cellular Structure of a TwoDimensional $\mathrm{H}_{2} / \mathrm{O}_{2} /$ Ar Detonation Wave," Combustion Theory and Modelling, Vol. 8, 2004, pp. 339-359. doi:10.1088/1364-7830/8/2/008

[55] Hu, X.Y., Zhang, D.L., Khoo, B.C., and Jiang, Z.L., "The Structure and Evolution of a TwoDimensional $\mathrm{H}_{2} / \mathrm{O}_{2} /$ Ar Cellular Detonation," Shock Waves, Vol. 14, No. 1-2, 2005, pp. 37-44. 
doi:0.1007/s00193-004-0234-5

[56] Strehlow R.A., "Gas Phase Detonations: Recent Developments," Combustion and Flame, Vol. 12, No. 2, 1968, pp. 81-101.

doi:10.1016/0010-2180(68)90083-7

[57] Goodwin, D., "Cantera: Object-Oriented Software for Reacting Flows," California Institute of Technology, http://www.cantera.org [retrieved 21 August 2015].

[58] Leer, B.V., "On the Relation between the Upwind-Differencing Schemes of Godunov, Engquist-Osher and Roe," SIAM Journal on Scientific and Statistical Computing, Vol. 5, No. 1, 1984, pp. 1-20. doi:10.1137/0905001

[59] Sharpe, G.J., "Transverse Waves in Numerical Simulations of Cellular Detonations," Journal of Fluid Mechanics, Vol. 447, 2001, pp. 31-51. doi:10.1017/S0022112001005535

[60] Samtaney, R., and Pullin, D.I., "On Initial-Value and Self-Similar Solutions of the Compressible Euler Equations," Physics of Fluids, Vol. 8, No. 10, 1996, pp. 2650-2655. doi:10.1063/1.869050

[61] Oran, E.S., Weber, J.W., Stefaniw, E.I., Lefebvre, M.H., and Anderson, J.D., “A Numerical Study of a Two-Dimensional $\mathrm{H}_{2}-\mathrm{O}_{2}$-Ar Detonation Using a Detailed Chemical Reaction Model," Combustion and Flame, Vol. 113, No. 1-2, 1998, pp. 147-163. doi:10.1016/S0010-2180(97)00218-6

[62] Mazaheri, K., Mahmoudi, Y., and Radulescu, M.I., "Diffusion and Hydrodynamic Instabilities in Gaseous Detonations," Combustion and Flame, Vol. 159, No. 6, 2012, pp. 2138-2154. 
doi:10.1016/j.combustflame.2012.01.024

[63] Mahmoudi, Y., Mazaherin, K., and Parvar, S., "Hydrodynamic Instabilities and Transverse Waves in Propagation Mechanism of Gaseous Detonations," Acta Astronautica, Vol. 91, 2013, pp. 263-282.

doi:10.1016/j.actaastro.2013.06.009

[64] Mahmoudi, Y., Karimi, N., Deiterding, R., and Emami, S., "Hydrodynamic Instabilities in Gaseous Detonations: Comparison of Euler, Navier-Stokes, and Large-Eddy Simulation," Journal of Propulsion and Power, Vol. 30, No. 2, 2014, pp. 384-396.

doi: $10.2514 / 1 . B 34986$

[65] Cai X.D., Liang, J.H., Deiterding, R., Che, Y.G., and Lin, Z.Y., “Adaptive Mesh Refinement Based Simulations of Three-Dimensional Detonation Combustion in Supersonic Combustible Mixtures with a Detailed Reaction Model,” International Journal of Hydrogen Energy, 2015. doi:10.1016/j.ijhydene.2015.11.093 